\title{
Amenable groups with very poor compression into Lebesgue spaces
}

\author{
Tim Austin
}

\begin{abstract}
We give a construction of finitely-generated amenable groups that do not admit any coarse 1-Lipschitz embedding with positive compression exponent into $L_{p}$ for any $1 \leq p<\infty$, including some that are four-step solvable, answering positively a question of Arzhantseva, Guba and Sapir.
\end{abstract}

\section{Contents}

1 Introduction 1

2 Background and generalities 6

2.1 Metric spaces . . . . . . . . . . . . . . . . . . . . . . . 6

2.2 Groups ........................ 8

3 Using distortion to bound compression 12

4 Construction of the example 15

4.1 Approximately equidistant sets . . . . . . . . . . . . . . 15

4.2 Some finite-dimensional quotient spaces . . . . . . . . . . . . . . 16

4.3 Completion of the proof . . . . . . . . . . . . 23

5 Further questions

\section{Introduction}

Given a metric space $(X, \rho)$ and a Banach space $\mathfrak{X}$ with norm $\|\cdot\|$, the compression exponent $\alpha_{\mathfrak{X}}^{*}(X, \rho)$ of $X$ into $\mathfrak{X}$ is the supremum of those $\alpha \geq 0$ for which there 
exists an injection $f: X \hookrightarrow \mathfrak{X}$ such that

$$
\rho(x, y)^{\alpha} \lesssim\|f(x)-f(y)\| \leq \rho(x, y) \quad \forall x, y \in X
$$

(where, as usual, $\lesssim$ and $\gtrsim$ denote inequalities that hold up to an arbitrary positive multiplicative constant that is independent of the arguments of the functions in question).

This can be viewed as a quantitative measure of how well, if at all, the space $(X, \rho)$ can be coarsely Lipschitzly embedded into $\mathfrak{X}$. It was introduced by Guentner and Kaminker in [10], with particular emphasis on the case when $(X, \rho)$ is a finitelygenerated group equipped with a left-invariant word metric associated to a finite symmetric generating set, and $\mathfrak{X}$ is a Hilbert space. In this case the value $\alpha_{\mathfrak{X}}^{*}(G)$ is easily seen to be independent of the particular choice of generating set, since different generating sets lead to quasi-isometric word metrics. For a group $G$ we can define analogously to $\alpha_{\mathfrak{X}}^{*}(G)$ the equivariant compression exponent $\alpha_{\mathfrak{X}}^{\#}(G)$ as the supremum of those $\alpha \geq 0$ for which there exists an equivariant injection $f: G \hookrightarrow \mathfrak{X}$ (that is, an injection given by the orbit of a point under some action $G \curvearrowright \mathfrak{X}$ by affine isometries) such that

$$
\rho(g, h)^{\alpha} \lesssim\|f(g)-f(h)\| \leq \rho(g, h) \quad \forall g, h \in G .
$$

We write $\alpha_{p}^{*}$ and $\alpha_{p}^{\#}$ in place of $\alpha_{L_{p}}^{*}$ and $\alpha_{L_{p}}^{\#}$. Since Guentner and Kaminker's work a number of methods have been brought to bear on the estimation of these exponents for different groups; see, for instance, [2, 3, 7, 8, 18, 19, 20, 17, 4, [15, 16]. A more detailed discussion of these developments and more complete references can be found in the introduction to [16]. It is also worth noting that if $G$ is amenable then necessarily $\alpha_{2}^{\#}(G)=\alpha_{2}^{*}(G)$, as was shown by Aharoni, Maurey and Mityagin in [1] for Abelian $G$ and then by Gromov (see [8]) in general.

Most known results concern the case of amenable groups. It was shown by Guentner and Kaminker in [10] that if $\alpha_{2}^{\#}(G)>\frac{1}{2}$ then this actually implies that $G$ is amenable, and another proof of this fact has now been given in [15] along with a generalization to other Lebesgue target spaces (although it is known that there are both amenable and non-amenable groups that have Euclidean equivariant compression exponent exactly equal to $\frac{1}{2}$ ). In the reverse direction, Arzhantseva, Guba and Sapir asked as Question 1.12 in [3] whether there are amenable groups that have compression exponent 0 (or, more modestly, strictly less than $\frac{1}{2}$ ) for embeddings into Hilbert space, and following progress in other directions versions of this question have since been re-posed as Question 5.23 of Arzhantseva, Drutu and Sapir [2] and Question 1.5 of Tessera [17]. 
This question is interesting partly because previous methods for bounding Hilbert space compression exponents from above seem unable in principle to break the $\frac{1}{2}$ barrier. In particular, a very general upper bound in terms of random walk escape speed, first introduced in [4] and then considerably generalized in [15, 16], cannot push below this value. One version of that result (although not quite the most general) asserts that if $\mathfrak{X}$ is a Banach space having modulus of smoothness of power type $p$, then $\alpha_{\mathfrak{X}}^{\#}(G) \leq \frac{1}{p \beta^{*}(G)}$, where $\beta^{*}(G)$ is the supremum of those $\beta \geq 0$ for which

$$
\mathrm{E}\left(\rho\left(e_{G}, X_{t}\right)\right) \gtrsim t^{\beta} \quad \forall t \in \mathbb{N},
$$

where $\left(X_{t}\right)_{t \geq 0}$ is the symmetric random walk on $G$ starting at the identity $e_{G}$ corresponding to some finite generating set. Since it is known that $\frac{1}{2} \leq \beta^{*} \leq 1$ always, this bound cannot give a value below $\frac{1}{2}$.

Moreover, in several concrete cases (in particular among certain iterated wreath products of cyclic groups) the random walk bound turns out to be the correct value of the compression exponent, even when this value lies in $\left(\frac{1}{2}, 1\right)$. These observations led Naor and Peres to ask in [16] (Question 10.3) whether it is always the case that an amenable group $G$ has compression exponent into $L_{p}$ given by exactly $\min \left\{\frac{1}{p \beta^{*}}, 1\right\}$ (which would, in particular, answer negatively the question of Arzhantseva, Guba and Sapir).

In this work we decide these questions by proving the following.

Theorem 1.1. There is a finitely-generated amenable group that does not admit any embedding into $L_{p}$ with a positive compression exponent for any $p \in[1, \infty)$.

Intuitively, this means we have found a finitely-generated amenable group with much worse embedding properties into $L_{p}$ than have been witnessed for such groups heretofore. Various notions relating to the uniform embeddability of finitelygenerated groups into 'nice' Banach spaces (predominantly Hilbert space) have been introduced in geometric group theory (see, in particular, Gromov's discussion of a-T-menability and some relatives in Section 7.E of [9]), and some equivalences found among them; on the other hand, some of these properties have been shown to have striking consequences elsewhere in gometric group theory and topology (perhaps most notably Yu's deduction of the coarse Baum-Connes and Novikov Conjectures therefrom in [21]). Gromov asked whether amenable groups are always a-T-menable, and this was then proved by Bekka, Cherix and Valette in [5]. Thus from the relatively soft viewpoint of coarse geometry amenable groups are known to possess such desirable embeddings; the present paper indicates that this is not a guarantee of any very strong quantitative versions of the same conclusions. 
Before launching into technical details, we offer a sketch of our approach to Theorem 1.1

Underpinning the proof is a simple observation about how a sequence of finite metric spaces with growing $L_{p}$-distortion can serve as an obstruction to the goodcompression $L_{p}$-embedding of an infinite metric space. If $(X, \rho)$ is an infinite metric space, and inside it we can find a family of bi-Lipschitzly embedded finite metric spaces $\left(Y_{n}, \sigma_{n}\right)$, say with embeddings $\varphi_{n}: Y_{n} \hookrightarrow X$, then these give rise to a bound on $\alpha_{p}^{*}(X, \rho)$ in terms of

- the distortions $c_{p}\left(Y_{n}, \sigma_{n}\right)$, and

- the ratios according to which distances under $\sigma_{n}$ are approximately expanded by $\varphi_{n}$.

This interplay between the distortions of the $Y_{n}$ and approximate expansion ratios of the $\varphi_{n}$ is crucial: the resulting obstruction to $L_{p}$-embedding of the whole of $(X, \rho)$ will be more severe according as the expansion ratios of these $\varphi_{n}$ grow more slowly. A quantitative version of this observation that is suitably tailored for our proof of Theorem 1.1 appears as Lemma 3.1 below. With this principle in hand, we will find the specific finite metric spaces that will serve as our obstructions in the form of certain quotients of high-dimensional Hamming cubes. Regarding Hamming cubes as vector spaces over $\mathbb{Z}_{2}$, a result of Khot and Naor [12] provides us with certain quotients of these by $\mathbb{Z}_{2}$-subspaces whose Euclidean distortion is linearly large in their diameter.

Most of our work will then go into constructing a group that contains suitable biLipschitz copies of these cube-quotients. The basic strategy of using a sequence of poor-distortion finite subsets to bound above the compression exponent of a group is not new; for example, Arzhantzeva, Druţu and Sapir use it in [2] for their construction of finitely-generated groups with arbitrary compression exponents into uniformly convex Banach spaces, except that their finitary obstructions are sequences of expanders, and they use free products (hence giving non-amenable examples) to construct finitely-generated groups containing them.

Our construction will give a two-fold Abelian extension of any suitable base group $G$ (which could be, for example, the classical lamplighter $\mathbb{Z}_{2} \imath \mathbb{Z}$, so that among our examples of groups with very poor compression we find certain four-step solvable groups). The first of these extensions of $G$ is a wreath product, but the second is something rather more complicated.

More precisely, we begin with any finitely-generated amenable $G$ that has expo- 
nential growth, form the wreath product over it $H:=\mathbb{Z}_{6} \succ G$ and equip it with its natural lifted generating set and word metric (we will comment on the modulus 6 shortly). Consider $H$ as acting by translation on the $\mathbb{Z}_{2}$-vector space $\mathbb{Z}_{2}^{\oplus H}$. These are the ingredients needed to form another wreath product $\mathbb{Z}_{2} \imath H=\mathbb{Z}_{2}^{\oplus H} \rtimes H$, but we will instead examine a slightly more complicated relative of this construction. (Indeed, while the random walk method gives an upper bound $\alpha_{2}^{*}\left(\mathbb{Z}_{2} \imath H\right) \leq \frac{1}{2}$, and a recent analysis by $\mathrm{Li}$ [13] building on a construction from de Cornulier, Stalder and Valette [6] has shown that it is at least $\frac{1}{6}$, its exact value is currently unknown. Arzhantseva, Guba and Sapir originally proposed the related iterated wreath product $\mathbb{Z} \imath(\mathbb{Z} \imath \mathbb{Z})$ as a candidate for having zero Euclidean compression exponent, but Li's work now also gives a positive lower bound for this exponent.) We will first identify a translation invariant $\mathbb{Z}_{2}$-subspace $V \leq \mathbb{Z}_{2}^{\oplus H}$, and will then form instead the semidirect product $\left(\mathbb{Z}_{2}^{\oplus H} / V\right) \rtimes H$, equipped with the natural generating set that consists of lifts of the generators of $H$ and the single new generator $\delta_{e_{H}}+V \leq \mathbb{Z}_{2}^{\oplus H} / V$.

The rôle of this $\mathbb{Z}^{2}$-subspace $V$ is to insert copies of a sequence of Khot and Naor's poor-distortion cube-quotients, say $\mathbb{Z}_{2}^{I_{n}} / C_{n}$ for some increasingly large index-sets $I_{1}, I_{2}, \ldots$, into the 'zero-section' subgroup

$$
\mathbb{Z}_{2}^{\oplus H} / V \subset\left(\mathbb{Z}_{2}^{\oplus H} / V\right) \rtimes H
$$

More precisely, we will prove that for some sequence of these cube-quotients $\mathbb{Z}_{2}^{I_{n}} / C_{n}$, one can effectively choose embeddings $\varphi_{n}^{\circ}: \mathbb{Z}_{2}^{I_{n}} \hookrightarrow \mathbb{Z}_{2}^{\oplus H}$ and a translationinvariant subgroup $V \leq \mathbb{Z}_{2}^{\oplus H}$ so that $\varphi_{n}^{\circ}\left(C_{n}\right) \subseteq V$, and the resulting quotient maps

$$
\varphi_{n}: \mathbb{Z}_{2}^{I_{n}} / C_{n} \hookrightarrow \mathbb{Z}_{2}^{\oplus H} / V \subset\left(\mathbb{Z}_{2}^{\oplus H} / V\right) \rtimes H
$$

are bi-Lipschitz embeddings with control on their distortions and expansion ratios as required for them to constrain the compression exponents of $L_{p}$-embeddings of the whole group $\left(\mathbb{Z}_{2}^{\oplus H} / V\right) \rtimes H$.

The principal difficulties in this construction arise from the need to insert a whole infinite sequence of these cube-quotients $\mathbb{Z}_{2}^{I_{n}} / C_{n}$ into $\mathbb{Z}_{2}^{\oplus H} / V$ using a single choice of subgroup $V$, with a uniform bound on their distortions and slow growth of their expansion ratios. It is to address these difficulties that we specifically take $H=\mathbb{Z}_{6} 2 G$ for some $G$ of exponential growth. The $\mathbb{Z}_{2}$-subspace $V$ will be assembled as an infinite sum $V_{1}+V_{2}+\ldots$ of $\mathbb{Z}_{2}$-subspaces, each of which creates the corresponding embedding of $\mathbb{Z}_{2}^{I_{n}} / C_{n}$, and we will use the particular algebraic structure of this wreath product $H$ to choose these $V_{n}$ so that they do not 'interact' with each other too much: more specifically, so that quotienting by $V_{n^{\prime}}$ for $n^{\prime}>n$ does not disrupt the bi-Lipschitz embedding of $\mathbb{Z}_{2}^{I_{n}} / C_{n}$ that we introduce upon 
quotienting by $V_{n}$. One feature of this algebraic argument is that it leads to the careful choice of 6 (rather than, say, 2 or 4 ) as the modulus in the wreath product $H=\mathbb{Z}_{6} 2 G$. This is used via the fact that there exists a non-constant function $\mathcal{A}: \mathbb{Z}_{6} \rightarrow \mathbb{Z}_{2}$ with the property that one cannot form the constant function $1 \in \mathbb{Z}_{2}$ as a $\mathbb{Z}_{2}$-linear comination of translates of $\mathcal{A}$ by elements of the domain $\mathbb{Z}_{6}$. For this we take $\mathcal{A}$ to be the $\mathbb{Z}_{2}$-valued indicator function of $\{0,1,3,4\}$. Since at a different step in the construction we will want a modulus that is even, a quick check shows that 6 is the smallest possibility. By allowing $\mathcal{A}$ to act on different coordinates in $\mathbb{Z}_{6}^{\oplus G}$ we can create a large family of functions $\mathbb{Z}_{6}^{\oplus G} \rightarrow \mathbb{Z}_{2}$ (that is, elements of

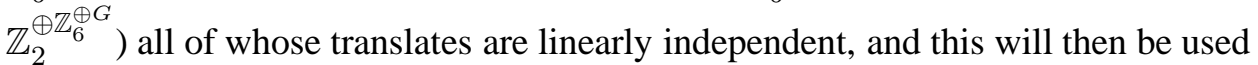
in the proof that our choices of translation invariant subspaces $V_{1}, V_{2}, \ldots \leq \mathbb{Z}_{2}^{\mathbb{Z}_{6} l G}$ are 'well-separated' (see Lemma 4.7 and its use in the proof of Proposition 4.5).

Acknowledgements I am grateful to Assaf Naor and Yuval Peres for introducing me to the topics touched by this paper and for many subsequent discussions, to Sean Li and Assaf Naor for each suggesting a number of improvements to this paper, and to Microsoft Research Redmond for a period of their hospitality during which most of this work was completed.

\section{Background and generalities}

\subsection{Metric spaces}

The following nomenclature distinguishing certain kinds of metric space will prove useful.

Definition 2.1. A metric space $(X, \rho)$ is

- infinite if this is so of $X$ as a set;

- locally finite if every open ball $B_{X}(x, r)$ in $X$ is a finite set;

- $d_{\min }$-discrete for some $d_{\min }>0$ if $\rho(x, y) \geq d_{\min }$ whenever $x, y \in X$ are distinct.

Given two metric spaces $(X, \rho)$ and $(Z, \theta)$ and a map $f: X \rightarrow Z$, the distortion of $f$ is a measure of the extent to which $f$ fails to be a homothety:

$$
\text { distortion }(f):=\sup _{u, v \in X, u \neq v} \frac{\theta(f(u), f(v))}{\rho(u, v)} \cdot \sup _{u, v \in X, u \neq v} \frac{\rho(u, v)}{\theta(f(u), f(v))},
$$


where this is interpreted as $+\infty$ unless $f$ is at least a bi-Lipschitz embedding. Relatedly, the map $f$ is $M$-bi-Lipschitz for some $M \geq 1$ if it is bi-Lipschitz with distortion at most $M$.

The distortion of $X$ into $Z$ is now obtained by infimizing over $f$ : it is conventionally denoted by

$$
c_{(Z, \theta)}(X, \rho):=\inf _{f: X \rightarrow Z} \operatorname{distortion}(f) .
$$

The case in which $(Z, \theta)$ is a Lebesgue space $L_{p}$ with its norm metric for some $1 \leq p<\infty$ is particularly well-studied, and in this case we abbreviate $c_{L_{p}}$ to $c_{p}$.

In addition to the distortion of a map $f$, we will sometimes need to keep track of its expansion ratio: in the above notation this is simply the ratio

$$
\inf _{u, v \in X, u \neq v} \frac{\theta(f(u), f(v))}{\rho(u, v)} .
$$

If the expansion ratio is $r$, then the above definitions combined tell us that

$$
r \cdot \rho(u, v) \leq \theta(f(u), f(v)) \leq \operatorname{distortion}(f) \cdot r \cdot \rho(u, v) \quad \forall u, v \in X,
$$

provided all the quantities appearing here are finite.

An important class of metrics that will appear repeatedly later is that of the Hamming metrics:

Definition 2.2 (Hamming metric). If $(X, \rho)$ is a metric space and $n \geq 1$ then the Hamming metric on $X^{n}$ associated to $\rho$ is defined by

$$
d_{\text {Ham }}\left(\left(x_{i}\right)_{i \leq n},\left(y_{i}\right)_{i \leq n}\right):=\sum_{i=1}^{n} \rho\left(x_{i}, y_{i}\right) .
$$

We will also make use of the construction of quotient metrics. The following definition is discussed, for example, in Section 3 of Khot and Naor [12].

Definition 2.3 (Quotient metrics). Suppose that $(X, \rho)$ is a metric space and that $\mathcal{P}$ is a partition of $X$ with the property that

For any $P, Q \in \mathcal{P}$ and any $x \in P$ there is some $y \in Q$ such that $\rho(x, y)=\operatorname{dist}_{\rho}(P, Q)$ (that is, 'the minimal distance between $P$ and $Q$ can be realized from any starting point on one side'). 
Then the quotient metric $\rho_{/ \mathcal{P}}$ on $\mathcal{P}$ is defined by

$$
\rho_{/ \mathcal{P}}(P, Q):=\operatorname{dist}_{\rho}(P, Q) .
$$

The routine verification that this is indeed a metric on $\mathcal{P}$ under the above assumption can be found in Section 3 of [12].

In particular, this definition always applies to give a metric on the homogeneous space $G / H$ when $(G, \rho)$ is a group carrying a left-invariant metric $\rho$ and $H \leq G$. In this case we denote the quotient metric by $\rho_{/ H}$.

Before leaving this subsection, let us recall some useful analyst's notation. Given positive quantities $A$ and $C$ and any other parameter $B$, we write $A \lesssim_{B} C$ or $A=$ $\mathrm{O}_{B}(C)$ if quantity $A$ is bounded above by quantity $C$ up to a positive multiplicative constant depending only on $B$ (and simply $A \lesssim C$ or $A=\mathrm{O}(C)$ if the constant is truly universal); similarly $A=\Omega_{B}(C)$ if $A$ is bounded from below by such a positive multiple of $C$; and $A=\Theta(C)$ if $A$ is bounded from above and below by the multiples of $C$ by two universal positive bounds (that is, if $A=\mathrm{O}(C)$ and $A=\Omega(C)$ ). Thus, for instance, an embedding has 'expansion ratio $\Theta(1)$ ' if its expansion can be shown to lie in $[r, R]$ for some $R \geq r>0$, independently of any other parameters that went into its construction.

\subsection{Groups}

The construction of this paper makes a double use of a semidirect product. Given a group $L$ and another $H$ that acts on it by automorphisms, $H \curvearrowright L$, we may form the semidirect product $H \ltimes L$ or $L \rtimes H$ as the set $L \times H$ equipped with the group operation

$$
\left(\ell_{1}, h_{1}\right) \cdot\left(\ell_{2}, h_{2}\right):=\left(\ell_{1}^{h_{2}} \cdot \ell_{2}, h_{1} h_{2}\right) .
$$

Importantly, the group $H \ltimes L$ is finitely-generated if

- $H$ is finitely-generated and

- $L$ admits a finite subset $S \subseteq L$ such that $L=\left\langle\left\{\alpha^{h}(s): h \in H, s \in S\right\}\right\rangle$.

This can be so even if $L$ itself is not finitely-generated. An important example of this is the wreath product $K \imath H:=K^{\oplus H} \rtimes H$, formed from the action $H \curvearrowright K^{\oplus H}$ (the group of $H$-indexed families of members of $K$ with cofinitely many entries equal to $e_{K}$ ) by coordinate right-translation so that

$$
\left(\left(k_{1, h}\right)_{h \in H}, h_{1}\right) \cdot\left(\left(k_{2, h}\right)_{h \in H}, h_{2}\right)=\left(\left(k_{1, h h_{2}^{-1}} k_{2, h}\right)_{h \in H}, h_{1} h_{2}\right) .
$$


This is always finitely-generated if this is so of $H$ and $K$, even though if $H$ is infinite then $L=K^{\oplus H}$ is not finitely-generated.

The semidirect product $L \rtimes H$ always contains a canonical copy of $L$ in the form of the subgroup $\left\{\left(\ell, e_{H}\right): \ell \in L\right\}$ : following Naor and Peres [16] we refer to this as the zero section of $L \rtimes H$ and sometimes denote it by $(L \rtimes H)_{0}$.

In this paper we will use a construction similar to that of the wreath product, except that the place of $K^{\oplus H}$ will sometimes be taken by one of its quotients. In the following the group $K$ will always be one of the cyclic groups $\mathbb{Z}_{2}$ or $\mathbb{Z}_{6}$. We will later form first an extension of a suitable base group by a power of $\mathbb{Z}_{6}$, and then a further extension by a power of $\mathbb{Z}_{2}$, and our choice of notation is geared to distinguish between these two extensions as clearly as possible. In particular:

- Given a base group $G$, we will usually denote elements of $\mathbb{Z}_{6}^{\oplus G}$ by lowercase bold letters such as $\mathbf{w}=\left(w_{g}\right)_{g \in G}$, and refer to them as vectors. In this setting we write $\mathbf{e}_{g}$ for $g \in G$ for the vector with $g^{\text {th }}$ entry equal to $1 \in$ $\mathbb{Z}_{6}$ and all other entries equal to 0 ; we refer to these $\mathbf{e}_{g}$ collectively as the standard generators of $\mathbb{Z}_{6}^{\oplus G}$.

- On the other hand, given another base group $H$ (which will later be equal to $\mathbb{Z}_{6}(G)$, we will denote elements of $\mathbb{Z}_{2}^{\oplus H}$ by uppercase calligraphic letters, and will refer to them as functions $\mathcal{W}: H \rightarrow \mathbb{Z}_{2}$; correspondingly an expression such as $\mathcal{W}+\mathcal{V}$ refers to a sum of functions defined pointwise. In this setting we denote by $\delta_{h}$ the function that takes the value $1 \in \mathbb{Z}_{2}$ at $h$ and 0 elsewhere. We will sometimes need to work instead with the set $\{h \in H: \mathcal{W}(h)=1\}$, which we refer to as the support of $\mathcal{W}$ and denote by $\operatorname{spt} \mathcal{W}$.

The only exceptions to these rules are that $\mathbf{0}$ will be used for the zero element in either case, since this should cause no confusion, and that for any finite index set $T$ we write $\mathbf{1}_{T}$ for the function $T \rightarrow C$ that identically takes the value $1 \in C$ for either $C=\mathbb{Z}_{2}$ or $C=\mathbb{Z}_{6}$.

If $V \leq \mathbb{Z}_{2}^{\oplus H}$ is a subspace that is invariant under the coordinate right-translation action of $H$, then this action of $H$ quotients to a well-defined action $H \curvearrowright \mathbb{Z}_{2}^{\oplus H} / V$. Given also a chosen finite symmetric generating set $S$ for $H$, we will always endow the semidirect product $\left(\mathbb{Z}_{2}^{\oplus H} / V\right) \rtimes H$ with the symmetric generating set

$$
\left\{\left(\delta_{e_{H}}+V, e_{H}\right)\right\} \cup\{(\mathbf{0}, s): s \in S\} .
$$

To this enlarged generating set we always associate the left-invariant word metric as usual. In general, if the generating set $S$ of $H$ is understood, the above generating 
set will also be understood for $\left(\mathbb{Z}_{2}^{\oplus H} / V\right) \rtimes H$, and we will (slightly abusively) denote the resulting metric by $\rho_{\left(\mathbb{Z}_{2}^{\oplus H} / V\right) \rtimes H}$, and will denote the restriction of this new metric to the zero section by $\rho_{\left(\left(\mathbb{Z}_{2}^{\oplus H} / V\right) \rtimes H\right)_{0}}$.

We will be working largely with the restriction of this word metric to the zero section of $\left(\mathbb{Z}_{2}^{\oplus H} / V\right) \rtimes H$, and to this end it will be helpful to have a simpler equivalent metric on the zero section to work with. Such a description can quite easily be given in terms of traveling salesman tours as a simple extension of the usual heuristic interpretation of the wreath product word metric in terms of lamplighter walks. The definition below has been adapted from Section 2 of Naor and Peres [16], where its connection with the problem of estimating the length of traveling salesman tours among points in a metric space (and, in particular, with the work of Jones in [11]) is explained in more detail.

Definition 2.4 (Pinned traveling salesman metrics). Given a metric space $(X, \rho)$ and a distinguished point $x^{\circ} \in X$, the associated traveling salesman metric pinned at $x_{0}$ is the metric $\operatorname{TS}_{\rho, x^{\circ}}$ on the collection $\mathbb{Z}_{2}^{\oplus X}$ of finitely-supported maps $X \rightarrow \mathbb{Z}_{2}$ (equivalently, finite subsets of $X$ ) defined by

$$
\begin{aligned}
& \operatorname{TS}_{\rho, x^{\circ}}(\mathcal{A}, \mathcal{B}) \\
& := \begin{cases}0 & \text { if } \mathcal{A}=\mathcal{B} \\
\min \left\{\begin{array}{c}
\sum_{i=1}^{\ell} \rho\left(x_{i}, x_{i+1}\right): \\
\text { the cycle }\left(x_{1}=x^{\circ}, x_{2}, \ldots, x_{\ell}, x_{\ell+1}=x^{\circ}\right) \\
\text { covers } \operatorname{spt}(\mathcal{A}+\mathcal{B})
\end{array}\right\}+1 & \text { else. }\end{cases}
\end{aligned}
$$

This is clearly a metric in view of the inclusion $\operatorname{spt}(\mathcal{A}+\mathcal{C}) \subseteq \operatorname{spt}(\mathcal{A}+\mathcal{B}) \cup \operatorname{spt}(\mathcal{B}+$ $\mathcal{C})$ and the ability to concatenate covering cycles.

Abbreviating, it will be understood that the notation $\mathrm{TS}_{\mathrm{Ham}}$ on $\mathbb{Z}_{2}^{\oplus \mathbb{Z}_{6}^{\oplus S}}$ refers to the Hamming metric $d_{\text {Ham }}$ on the underlying group $\mathbb{Z}_{6}^{\oplus S}$ (where $\mathbb{Z}_{6}$ is given the word metric corresponding to the generators $\{1,-1\})$ and the distinguished point $x^{\circ}:=\mathbf{0} \in \mathbb{Z}_{6}^{\oplus S}$.

If $T$ is a finite index set and $F \leq \mathbb{Z}_{2}^{\mathbb{Z}_{6}^{T}}$ is a $\mathbb{Z}_{2}$-subspace invariant under the translation action of $\mathbb{Z}_{6}^{T}$, then we denote by $\mathrm{TS}_{\mathrm{Ham} / F}$ the metric on $\mathbb{Z}_{2}^{\mathbb{Z}_{6}^{T}} / F$ that results from quotienting $\mathrm{TS}_{\mathrm{Ham}}$.

Let us note at once that following trivial consequence of this definition:

Lemma 2.5. If $\mathcal{V}, \mathcal{V}^{\prime}, \mathcal{W}, \mathcal{W}^{\prime}: X \rightarrow \mathbb{Z}_{2}$ are finitely supported and $x^{\circ} \in X$ then

$$
\operatorname{spt}\left(\mathcal{V}^{\prime}+\mathcal{W}^{\prime}\right) \supseteq \operatorname{spt}(\mathcal{V}+\mathcal{W}) \quad \Rightarrow \quad \operatorname{TS}_{\rho, x^{\circ}}\left(\mathcal{V}^{\prime}, \mathcal{W}^{\prime}\right) \geq \operatorname{TS}_{\rho, x^{\circ}}(\mathcal{V}, \mathcal{W}) .
$$


In particular, $\mathrm{TS}_{\rho, x^{\circ}}$ is addition-invariant on $\mathbb{Z}_{2}^{\oplus X}$.

A more delicate calculation that we will need later is the following. This closely resembles the derivation of equation (13) in Naor and Peres [16], and we offer only a rather terse account here.

Lemma 2.6. If $\left(\mathbb{Z}_{2}^{\oplus H} / V\right) \rtimes H$ is a semidirect product as above, $H$ has generating set $S$ and corresponding word metric $\rho$ and we lift this to a generating set of $\left(\mathbb{Z}_{2}^{\oplus H} / V\right) \rtimes H$ as above with associated metric $\rho_{\left(\mathbb{Z}_{2}^{\oplus H} / V\right) \rtimes H}$, then the inclusion $\mathbb{Z}_{2}^{\oplus H} / V \equiv\left(\left(\mathbb{Z}_{2}^{\oplus H} / V\right) \rtimes H\right)_{0} \subset\left(\mathbb{Z}_{2}^{\oplus H} / V\right) \rtimes H$ is a 2-bi-Lipschitz embedding of $\mathrm{TS}_{\rho, e_{H} / V}$ into $\rho_{\left(\mathbb{Z}_{2}^{\oplus H} / V\right) \rtimes H}$ with expansion ratio lying in $\Theta(1)$.

To find a shortest-length word that evaluates to a given group element $\left(\mathcal{A}+V, e_{H}\right) \in$ $\left(\mathbb{Z}_{2}^{\oplus H} / V\right) \rtimes H$ amounts to finding a shortest walk from $\left(V, e_{H}\right)$ to $\left(\mathcal{A}+V, e_{H}\right)$ that steps only along edges of the Cayley graph defined by the generated set of $\left(\mathbb{Z}_{2}^{\oplus H} / V\right) \rtimes H$. Intuitively, in order to do this we must take a walk in the Cayley graph of $H$ that starts and ends at $e_{H}$, and along the way pass through finitely many points of $H$ at which we modify the value taken by the identically-zero function $0: H \rightarrow \mathbb{Z}_{2}$ to obtain a function that lies in $\mathcal{A}+V$ (this much corresponds roughly to an evaluation of a distance in $\operatorname{TS}_{\rho, e_{H}}$ ), and finally infimize over the choice of that element of $\mathcal{A}+V$ (which corresponds to working in the quotient metric $\mathrm{TS}_{\rho, e_{H} / V}$ ). The following proof makes this intuition precise.

Proof We will show that for any finitely-supported $\mathcal{A}, \mathcal{B}: H \rightarrow \mathbb{Z}_{2}$ we have

$$
\begin{aligned}
\operatorname{TS}_{\rho, e_{H} / V}(\mathcal{A}+V, \mathcal{B}+V) \leq \rho_{\left(\mathbb{Z}_{2}^{\oplus H} / V\right) \rtimes H}( & \left.\left(\mathcal{A}+V, e_{H}\right),\left(\mathcal{B}+V, e_{H}\right)\right) \\
\leq 2 \cdot \operatorname{TS}_{\rho, e_{H}} / V & (\mathcal{A}+V, \mathcal{B}+V) .
\end{aligned}
$$

To see this, recall that by definition $\rho_{\left(\mathbb{Z}_{2}^{\oplus H} / V\right) \rtimes H}\left(\left(\mathcal{A}+V, e_{H}\right),\left(\mathcal{B}+V, e_{H}\right)\right)$ is the shortest length of any word in the alphabet given by the generating set

$$
\left\{\left(\delta_{e_{H}}+V, e_{H}\right)\right\} \cup\{(V, s): s \in S\}
$$

whose evaluation in $\left(\mathbb{Z}_{2}^{\oplus H} / V\right) \rtimes H$ is equal to $\left(\mathcal{A}+V, e_{H}\right)^{-1} \cdot\left(\mathcal{B}+V, e_{H}\right)=$ $\left(\mathcal{B}-\mathcal{A}+V, e_{H}\right)$. Set $\ell:=\rho_{\left(\mathbb{Z}_{2}^{\oplus H} / V\right) \rtimes H}\left(\left(\mathcal{A}+V, e_{H}\right),\left(\mathcal{B}+V, e_{H}\right)\right)$, let $g_{\ell} g_{\ell-1} \cdots g_{1}$ be such a word of minimal length in this alphabet and define $G_{i}:=g_{i} g_{i-1} \cdots g_{1}=$ : $\left(\mathcal{C}_{i}+V, h_{i}\right)$ for $i=1,2, \ldots, \ell$ and also $G_{0}:=\left(V, e_{H}\right)=:\left(\mathcal{C}_{0}+V, h_{0}\right)$.

Each $G_{i}$ differs from $G_{i-1}$ by left-multiplication by either $\left(\delta_{e_{H}}+V, e_{H}\right)$ or by $(V, s)$ for some $s \in S$. In the first case the multiplication rule (1) tells us that 
$h_{i}=h_{i-1}$ and $\mathcal{C}_{i}=\mathcal{C}_{i-1}+\delta_{h_{i-1}} \bmod V$, and in the second it gives $\mathcal{C}_{i}=\mathcal{C}_{i-1}$ but $h_{i}=g_{i} h_{i-1}$. Letting $1 \leq i_{1}<i_{2}<\ldots<i_{k} \leq \ell$ be the subsequence of those $i$ at which we are in the first of these situations, we see that overall

$$
\mathcal{C}_{\ell}=\delta_{h_{i_{1}-1}}+\delta_{h_{i_{2}-1}}+\cdots+\delta_{h_{i_{k}-1}}=\mathcal{A}-\mathcal{B} \bmod V .
$$

Therefore, omitting the steps $i_{1}, i_{2}, \ldots, i_{k}$, the sequence $h_{1}, h_{2}, \ldots, h_{i_{1}-1}, h_{i_{1}+1}$, $\ldots, h_{\ell}$ executes a walk in $H$ that starts from $e_{H}$, visits every point of spt $\mathcal{C}_{\ell}$ (where $\left.\mathcal{C}_{\ell} \in \mathcal{A}-\mathcal{B}+V\right)$ at least once and then returns to $e_{H}$; and at those omitted steps the multiplication by $g_{i_{j}}$ does not change the position of $h_{i_{j}-1}$ but instead alters the function $\mathcal{C}_{i_{j}-1}$ to $\mathcal{C}_{i_{j}}$ modulo $V$.

On the one hand, it follows immediately from Definition 2.4 that the length of this walk is at least $\operatorname{TS}_{\rho, e_{H}}\left(\mathbf{0}, \mathcal{C}_{\ell}\right)$; and on the other, for any such $\mathcal{C}_{\ell}$, since any two points of $\operatorname{spt} \mathcal{C}_{\ell}$ are separated by a distance of at least 1 in $H$ under $\rho$, it follows that there does exist such a sequence of walk-steps interspersed with additions of $\delta_{h_{i_{k}}}+V$ of length at most

$$
\operatorname{TS}_{\rho, e_{H}}\left(\mathbf{0}, \mathcal{C}_{\ell}\right)+\left|\mathcal{C}_{\ell}\right| \leq 2 \cdot \operatorname{TS}_{\rho, e_{H}}\left(\mathbf{0}, \mathcal{C}_{\ell}\right)
$$

Therefore, first minimizing the above lower bound over the possible choices of $\mathcal{C}_{\ell} \in \mathcal{A}-\mathcal{B}+V$ we obtain

$$
\begin{aligned}
\operatorname{TS}_{\rho, e_{H} / V}(\mathcal{A}+V, \mathcal{B}+V)= & \min _{\mathcal{C} \in \mathcal{A}-\mathcal{B}+V} \operatorname{TS}_{\rho, e_{H}}(\mathbf{0}, \mathcal{C}) \\
& \leq \rho_{\left(\mathbb{Z}_{2}^{\oplus H} / V\right) \rtimes H}\left(\left(\mathcal{A}+V, e_{H}\right),\left(\mathcal{B}+V, e_{H}\right)\right)
\end{aligned}
$$

and secondly choosing $\mathcal{C}=\mathcal{C}_{\ell}$ that attains this minimum and then selecting a suitable word $g_{\ell} g_{\ell-1} \cdots g_{1}$ as described above proves the corresponding upper bound.

\section{Using distortion to bound compression}

Before turning to the construction of our group, we examine the relation between the notions of compression for infinite metric spaces and of distortion for finite metric spaces. In particular, we will see how to use large high-distortion finite subsets of an infinite metric space as obstructions to good-compression embeddings of the whole space, in the sense made precise by the following. 
Lemma 3.1. Suppose that $\mathfrak{X}$ is a normed vector space, that $(X, \rho)$ is an infinite, locally finite, 1-discrete metric space, and suppose further that we can find a sequence of finite 1-discrete metric spaces $\left(Y_{n}, \sigma_{n}\right)$ and embeddings $\varphi_{n}: Y_{n} \hookrightarrow X$ such that

- the $Y_{n}$ are increasing in diameter: $\operatorname{diam}\left(Y_{n}, \sigma_{n}\right) \rightarrow \infty$;

- the $Y_{n}$ are embedded in $X$ with uniformly-bounded distortion: there are some fixed $L \geq 1$ and some sequence of positive reals $\left(r_{n}\right)_{n \geq 1}$ such that

$$
\frac{1}{L} r_{n} \sigma_{n}(u, v) \leq \rho\left(\varphi_{n}(u), \varphi_{n}(v)\right) \leq L r_{n} \sigma_{n}(u, v) \quad \forall u, v \in Y_{n}, n \geq 1
$$

(so the distortion is at most $L^{2}$ );

- the $Y_{n}$ do not expand too fast inside $X$ relative to their size: we have $1 \leq$ $r_{n} \lesssim \operatorname{diam}\left(Y_{n}, \sigma_{n}\right)^{\varepsilon}$ for every $\varepsilon>0$

- the $Y_{n}$ have bad distortion into $\mathfrak{X}$ : for some $\eta>0$ we have $c_{\mathfrak{X}}\left(Y_{n}, \sigma_{n}\right) \gtrsim$ $\operatorname{diam}\left(Y_{n}, \sigma_{n}\right)^{\eta}$ for all $n \geq 1$.

Then $\alpha_{\mathfrak{X}}^{*}(X, \rho) \leq 1-\eta$.

Proof We may assume that $\alpha^{*}(X, \rho)>0$, since otherwise the result is trivial. Given this, let $\alpha<\alpha^{*}(X, d)$ and let $f: X \rightarrow \mathfrak{X}$ be a 1-Lipschitz embedding into $\mathfrak{X}$ that achieves compression $\alpha$ :

$$
\rho(x, y)^{\alpha} \lesssim\|f(x)-f(y)\| \leq \rho(x, y) \quad \forall x, y \in X .
$$

Combining this with the bi-Lipschitz condition

$$
\frac{1}{L} r_{n} \sigma_{n}(u, v) \leq \rho\left(\varphi_{n}(u), \varphi_{n}(v)\right) \leq L r_{n} \sigma_{n}(u, v)
$$

by setting $x:=\varphi_{n}(u)$ and $y:=\varphi_{n}(v)$ in the compression inequality, we deduce that $f \circ \varphi_{n}: Y_{n} \hookrightarrow \mathfrak{X}$ satisfies

$$
\frac{1}{L^{\alpha}}\left(r_{n} \sigma_{n}(u, v)\right)^{\alpha} \lesssim\left\|f \circ \varphi_{n}(u)-f \circ \varphi_{n}(v)\right\| \leq L r_{n} \sigma_{n}(u, v) \quad \forall u, v \in Y_{n}, n \geq 1 .
$$

It follows that

$$
\begin{aligned}
& \text { distortion }\left(f \circ \varphi_{n}\right) \\
& \stackrel{\text { dfn }}{=} \max _{u, v \in Y_{n}, u \neq v} \frac{\sigma_{n}(u, v)}{\left\|f \circ \varphi_{n}(u)-f \circ \varphi_{n}(v)\right\|} \cdot \max _{u, v \in Y_{n}, u \neq v} \frac{\left\|f \circ \varphi_{n}(u)-f \circ \varphi_{n}(v)\right\|}{\sigma_{n}(u, v)} \\
& \lesssim\left(\max _{u, v \in Y_{n}, u \neq v} L^{\alpha} r_{n}^{-\alpha} \sigma_{n}(u, v)^{1-\alpha}\right) \cdot L r_{n} \\
& =L^{1+\alpha} r_{n}^{1-\alpha} \operatorname{diam}\left(Y_{n}, \sigma_{n}\right)^{1-\alpha} .
\end{aligned}
$$


Since we know that this must be at least $c_{\mathfrak{X}}\left(Y_{n}, \sigma_{n}\right) \gtrsim \operatorname{diam}\left(Y_{n}, \sigma_{n}\right)^{\eta}$, it follows that

$$
\operatorname{diam}\left(Y_{n}, \sigma_{n}\right)^{\eta} \lesssim L^{1+\alpha} r_{n}^{1-\alpha} \operatorname{diam}\left(Y_{n}, \sigma_{n}\right)^{1-\alpha}
$$

Finally, from our assumption that $r_{n} \lesssim \operatorname{diam}\left(Y_{n}, \sigma_{n}\right)^{\varepsilon}$ for every $\varepsilon>0$ and since $L$ is independent of $n$, it follows that

$$
\operatorname{diam}\left(Y_{n}, \sigma_{n}\right)^{\eta} \lesssim \operatorname{diam}\left(Y_{n}, \sigma_{n}\right)^{(1-\alpha)(1+\varepsilon)} \quad \text { as } n \rightarrow \infty
$$

for every $\varepsilon>0$, and hence that $1-\alpha \geq \eta$, or $\alpha \leq 1-\eta$.

Remark Note that the interplay between the expansion ratio $r_{n}$ incurred by the embedding $\varphi_{n}: Y_{n} \hookrightarrow X$ and the compression assumption on $f$ is crucial for this proof: if, in a different situation, we knew that we could embed the same metric spaces $Y_{n}$ into $X$ bi-Lipschitzly, but only at the expense of drastically enlarging them, then the above argument would give no bound on the compression.

In conjunction with the general principle contained in the above lemma, we will use the following result of Khot and Naor [12] to supply us with high-distortion finite metric spaces to serve as obstructions.

Theorem 3.2. There are $\mathbb{Z}_{2}$-subspaces $V_{d} \leq \mathbb{Z}_{2}^{d}$ for all $d \geq 1$ such that

$$
c_{p}\left(\mathbb{Z}_{2}^{d} / V_{d}, d_{\mathrm{Ham} / V_{d}}\right) \gtrsim_{p} d
$$

as $d \rightarrow \infty$ for all $p \in[1, \infty)$. Moreover, the subspace $V_{d}$ may be taken of the form $C_{d}^{\perp}$ for some other subspace $C_{d} \leq \mathbb{Z}_{2}^{d}$ such that $\mathbf{1}=(1,1, \ldots, 1) \in C_{d}$, where

$$
C_{d}^{\perp}:=\left\{\left(w_{i}\right)_{i \leq d} \in \mathbb{Z}_{2}^{d}: w_{1} v_{1}+w_{2} v_{2}+\cdots+w_{d} v_{d}=0 \in \mathbb{Z}_{2} \forall\left(v_{i}\right)_{i \leq d} \in C_{d}\right\} .
$$

Proof The existence of such subspaces is covered directly by Remark 3.1 in [12], and the fact that we may take $\mathbf{1} \in C_{d}$ follows at once from the greedy-algorithm proof given for their Corollary 3.5.

With these ingredients to hand, we can now state a more precise result from which Theorem 1.1 will follow immediately.

Theorem 3.3. For any finitely-generated amenable group $G$ of exponential growth, with finite symmetric generating set $S$ and associated left-invariant word metric $\rho$, there are a fixed $M \geq 1$ and $a \mathbb{Z}_{2}$-subspace $V \leq \mathbb{Z}_{2}^{\left.\oplus\left(\mathbb{Z}_{6}\right\} G\right)}$ such that

- $V$ is invariant under the action of $\mathbb{Z}_{6} 2 G$ by coordinate right-translation, and 
- the natural choice of generating set for $\left(\mathbb{Z}_{2}^{\oplus\left(\mathbb{Z}_{6} \imath G\right)} / V\right) \rtimes\left(\mathbb{Z}_{6} \zeta G\right)$ admits a sequence of injections

$$
\varphi_{n}: \mathbb{Z}_{2}^{d_{n}} / V_{d_{n}} \hookrightarrow \mathbb{Z}_{2}^{\oplus\left(\mathbb{Z}_{6} \imath G\right)} / V \subset\left(\mathbb{Z}_{2}^{\oplus\left(\mathbb{Z}_{6} \imath G\right)} / V\right) \rtimes\left(\mathbb{Z}_{6} \curlywedge G\right)
$$

that are embeddings of $d_{\mathrm{Ham} / V_{d_{n}}}$ into $\rho_{\left(\mathbb{Z}_{2}^{\oplus\left(\mathbb{Z}_{6} \imath G\right)} / V\right) \rtimes\left(\mathbb{Z}_{6} \imath G\right)}$ with uniformlybounded distortions and expansion ratios $r_{n} \lesssim \log d_{n}$.

Proof of Theorem 1.1 from Theorem 3.3 This now follows by applying Lemma3.1 using the Euclidean distortion bound from Theorem 3.2 according to which we can take $\eta=1$ when the spaces $\left(Y_{n}, \sigma_{n}\right)$ are the cube-quotients $\left(\mathbb{Z}_{2}^{d_{n}} / V_{d_{n}}, d_{\mathrm{Ham} / V_{d_{n}}}\right)$.

The rest of this paper will be taken up by the proof of Theorem 3.3. All the delicacy in this construction lies in the selection of the subspace $V$. The formation of a tower of two semidirect products (starting from a base group $G$ of exponential growth, which could also be obtained as another semidirect product) is rather important for the detailed arguments to come, and leads to examples, at their simplest, that are 4-step solvable. Note that they are, at least, elementary amenable. The ways in which we use each of these semidirect products, and also the reasons for choosing the particular cyclic groups $\mathbb{Z}_{2}$ and $\mathbb{Z}_{6}$ as building blocks, will be examined as they arise during the subsequent sections.

\section{Construction of the example}

\subsection{Approximately equidistant sets}

Our first step in the construction of the subspace $V$ promised by Theorem 3.3 is the following.

Lemma 4.1 (Finding not-too-wide sets of many roughly equidistant points). Let $G$ be a finitely-generated group of exponential growth, $S$ a symmetric generating set and $\rho$ the resulting word metric. Then there are some $M \geq 1$ and $c>1$, depending only on $G$ and $S$, such that for any $r_{0}>0$ there is some $r \geq r_{0}$ for which we can find a subset $\left\{x_{1}, x_{2}, \ldots, x_{d}\right\}$ with $d:=10^{r}$ such that $r \leq \rho\left(x_{i}, x_{j}\right) \leq 2 M r$ for all $1 \leq i<j \leq d$ and $r \leq \rho\left(x_{i}, e_{G}\right) \leq 2 M r$ for all $1 \leq i \leq d$.

Proof Let $c>1$ be such that

$$
|B(e, r)| \geq c^{r} \quad \text { for infinitely many } r \geq 1 .
$$


In the reverse direction, a naïve count tells us that

$$
|B(e, r)| \leq|S|^{r} \quad \forall r \geq 1 .
$$

Let $M \geq 1$ be a fixed integer such that $c^{M}>(11|S|)^{2}$. Then for any $r_{0} \geq 1$ we can find some $r \geq r_{0}+1$ and $0 \leq s \leq M-1$ such that

$$
|B(e, M r)| \geq|B(e, M(r-1)+s)| \geq\left(c^{M}\right)^{r-1} \cdot c^{s} \geq(11|S|)^{2(r-1)} \geq 11^{r} \cdot|S|^{r},
$$

and the above inequalities together now imply that

$$
|B(e, M r) \backslash B(e, r)|=|B(e, M r)|-|B(e, r)| \geq\left(11^{r}-1\right) \cdot|S|^{r} \geq 10^{r} \cdot|S|^{r} .
$$

Using again that $|B(x, r)| \leq|S|^{r}$ for every $(x, r)$, it follows that $B(e, M r) \backslash$ $B(e, r)$ cannot be covered by fewer than $10^{r}$ balls of radius $r$. This implies instead that we can find in $B(e, M r) \backslash B(e, r)$ a subset containing at least $d:=10^{r}$ points, any two of which are separated by a distance of at least $r$. Enumerating this subset as $\left\{x_{1}, x_{2}, \ldots, x_{d}\right\}$ completes the proof.

\subsection{Some finite-dimensional quotient spaces}

By considering the subsets

$$
\left\{\left(\sum_{i=1}^{d} w_{i} \mathbf{e}_{x_{i}}, e_{G}\right):\left(w_{i}\right)_{i \leq d} \in\{0,1\}^{d}\right\} \subset\left(\mathbb{Z}_{6} \succ G\right)_{0}
$$

with $\left\{x_{1}, x_{2}, \ldots, x_{d}\right\}$ as given by Lemmas 4.1, it is not hard to deduce from Lemma 2.6 how to embed copies of the Hamming cubes $\mathbb{Z}_{2}^{d}$ with uniform distortion inside $\mathbb{Z}_{6} 2 G$ so that their diameters grow exponentially faster than the expansion ratios of their embeddings. However, to obtain cube-quotients requires a little more work, in this subsection we prepare the ground for this.

The difficulty that seems to prevent us from finding these quotients inside $\mathbb{Z}_{6}$ $2 G$ lies in finding a single subgroup of $\mathbb{Z}_{6}^{\oplus G}$ that is $G$-translation invariant and for which the resulting quotient will restrict to the desired quotient on each of these Hamming cube copies separately. It is for this reason that we make a second extension of our group, to form our final group of the form $\left(\mathbb{Z}_{2}^{\oplus\left(\mathbb{Z}_{6} \zeta G\right)} / V\right) \rtimes\left(\mathbb{Z}_{6} \zeta G\right)$ for a suitable choice of $\left(\mathbb{Z}_{6}, G\right)$-translation invariant $\mathbb{Z}_{2}$-subspace $V$. Even in this case we will need to take some care in our proof that the quotient by $V$ really does lead to the bi-Lipschitz obstructions we seek. 
In this subsection we begin by putting the finite-dimensional quotient spaces of Theorem 3.2 into a form that is better adapted to embedding them into a leftinvariant group metric.

First, by an iterated appeal to Lemma 4.1 we can clearly select an increasing sequence of positive reals $\left(r_{n}\right)_{n \geq 1}$, a sequence of points $\left(y_{n}\right)_{n \geq 1}$ in $G$ and a sequence of pairwise-disjoint subsets $\left(I_{n}\right)_{n \geq 1}$ of $G$ such that

- $I_{n} \subset G \backslash\left\{y_{1}, y_{2}, \ldots\right\}$ for all $n \geq 1$,

- $r_{n} \leq \rho\left(x, x^{\prime}\right) \leq 2 M r_{n}$ for all distinct $x, x^{\prime} \in I_{n}$ and $r_{n} \leq \rho\left(x, e_{G}\right) \leq$ $2 M r_{n}$ for all $x \in I_{n}, n \geq 1$,

- $\left|I_{n}\right|=d_{n}:=10^{r_{n}}$ for all $n \geq 1$, and

- we have

$$
r_{1}<\rho\left(y_{1}, e_{G}\right)<r_{2}<\rho\left(y_{2}, e_{G}\right)<\ldots
$$

It will matter at various points later that the sequence of positive reals $r_{1}, \rho\left(y_{1}, e_{G}\right)$, $r_{2}, \rho\left(y_{2}, e_{G}\right), \ldots$ grows sufficiently quickly. However, in the remainder of this subsection we will consider only the data of a single set $I_{n}$.

From this set we form the group $\mathbb{Z}_{6}^{I_{n}}$. This is a finite-dimensional factor of $\mathbb{Z}_{6}^{\oplus G} \cong$ $\left(\mathbb{Z}_{6}<G\right)_{0}$. We endow it with the Hamming metric $d_{\text {Ham }}$ that arises from endowing each factor copy of $\mathbb{Z}_{6}$ separately with the word metric corresponding to the generating set $\{ \pm 1\}$.

Within this group we will consider the subset $B_{n}:=\left\{\mathbf{e}_{x}: x \in I_{n}\right\}$ comprising the standard set of basis vectors. Let $C_{n} \leq \mathbb{Z}_{2}^{I_{n}}$ be some $\mathbb{Z}_{2}$-subspace as appears in the Theorem 3.2, so that the cube-quotient $\mathbb{Z}_{2}^{I_{n}} / C_{n}^{\perp}$ endowed with the quotient of the Hamming metric has $L_{p}$-distortion $\Omega_{p}\left(d_{n}\right)$ for all finite $p$.

The space $\mathbb{Z}_{2}^{I_{n}}$ embeds into $\mathbb{Z}_{2}^{\mathbb{Z}_{6}^{I_{n}}}$ through its identification with $\mathbb{Z}_{2}^{B_{n}}$ and thence with

$$
\left.\left\{\mathcal{W}: \mathbb{Z}_{6}^{I_{n}} \rightarrow \mathbb{Z}_{2}: \text { spt } \mathcal{W} \subseteq B_{n}\right\} \cong \mathbb{Z}_{2}^{B_{n}} \oplus \mathbf{0}\right|_{\mathbb{Z}_{6}^{I_{n}} \backslash B_{n}} ;
$$

let us write $\xi: \mathbb{Z}_{2}^{I_{n}} \hookrightarrow \mathbb{Z}_{2}^{\mathbb{Z}_{6}^{I_{n}}}$ for this embedding. In order to avoid confusion we denote by

$$
\langle\mathbf{a}, \mathbf{b}\rangle:=\sum_{x \in I_{n}} a_{x} b_{x} \in \mathbb{Z}_{2}
$$


the $\mathbb{Z}_{2}$-valued inner product on $\mathbb{Z}_{2}^{I_{n}}$, and by

$$
\langle\langle\mathcal{W}, \mathcal{V}\rangle\rangle:=\sum_{\mathbf{w} \in \mathbb{Z}_{6}^{I_{n}}} \mathcal{W}(\mathbf{w}) \mathcal{V}(\mathbf{w}) \in \mathbb{Z}_{2}
$$

its analog on the space $\mathbb{Z}_{2}^{\mathbb{Z}_{6}^{I_{n}}}$ of functions.

We will now show how to convert $C_{n}$ into a $\mathbb{Z}_{2}$-subspace $D_{n} \leq \mathbb{Z}_{2}^{\mathbb{Z}_{6}^{I_{n}}}$ that is

- invariant under the translation action of $\mathbb{Z}_{6}^{I_{n}}$, and

- such that the restriction of $\mathrm{TS}_{\mathrm{Ham} / D_{n}^{\perp}}$ to

$$
\left\{\mathcal{V}+D_{n}^{\perp}:\left.\mathcal{V} \in \mathbb{Z}_{2}^{B_{n}} \oplus \mathbf{0}\right|_{\mathbb{Z}_{6}^{I_{n}} \backslash B_{n}}\right\}
$$

is equivalent under $\xi$ to the quotient of $d_{\mathrm{Ham}}$ on $\mathbb{Z}_{2}^{I_{n}} / C_{n}^{\perp}$.

These conclusions will be a corollary of the following lemma.

Lemma 4.2. There is a subspace $D_{n} \leq \mathbb{Z}_{2}^{\mathbb{Z}_{6}^{I_{n}}}$ that is

- invariant under the translation action of $\mathbb{Z}_{6}^{I_{n}}$,

- such that

$$
D_{n}^{\perp} \cap\left(\left.\mathbb{Z}_{2}^{B_{n}} \oplus \mathbf{0}\right|_{\mathbb{Z}_{6}^{I_{n}} \backslash B_{n}}\right)=\left.\xi\left(C_{n}^{\perp}\right) \oplus \mathbf{0}\right|_{\mathbb{Z}_{6}^{I_{n}} \backslash B_{n}},
$$

- and such that whenever $\mathcal{V}, \mathcal{V}^{\prime}: \mathbb{Z}_{6}^{I_{n}} \rightarrow \mathbb{Z}_{2}$ are supported on $B_{n}$, the distance

$$
\operatorname{TS}_{\mathrm{Ham} / D_{n}^{\perp}}\left(\mathcal{V}+D_{n}^{\perp}, \mathcal{V}^{\prime}+D_{n}^{\perp}\right)
$$

is attained as $\operatorname{TS}_{\mathrm{Ham}}(\mathcal{V}, \mathcal{W})$ for some $\mathcal{W} \in \mathcal{V}^{\prime}+D_{n}^{\perp}$ that is also supported on $B_{n}$ (and hence, by the second point above, is identified with the sum of $\mathcal{V}^{\prime}$ and a member of $\xi\left(C_{n}^{\perp}\right)$ ).

Proof Given $\mathbf{w} \in \mathbb{Z}_{6}^{T}$ for any index set $T$, let $\overline{\mathbf{w}} \in \mathbb{Z}_{2}^{T}$ be its image under the coordinatewise application of the quotient homomorphism $\mathbb{Z}_{6} \rightarrow \mathbb{Z}_{2}$.

Now, for $\mathbf{a}=\left(a_{x}\right)_{x} \in \mathbb{Z}_{2}^{I_{n}}$ we define the associated linear functional $\mathcal{L}_{\mathbf{a}}: \mathbb{Z}_{6}^{I_{n}} \rightarrow$ $\mathbb{Z}_{2}$ by composition with this quotient homomorphism and duality:

$$
\mathcal{L}_{\mathbf{a}}(\mathbf{v})=\langle\overline{\mathbf{v}}, \mathbf{a}\rangle=\sum_{x \in I_{n}} \overline{v_{x}} a_{x} .
$$


Given $C_{n}$ from Theorem 3.2, we simply let $D_{n}:=\left\{\eta+\mathcal{L}_{\mathbf{a}}: \eta \in \mathbb{Z}_{2}, \mathbf{a} \in C_{n}\right\}$. This collection of maps $\mathbb{Z}_{6}^{I_{n}} \rightarrow \mathbb{Z}_{2}$ is clearly a $\mathbb{Z}_{2}$-subspace by the $\mathbb{Z}_{2}$-linearity of $\mathbf{a} \mapsto \mathcal{L}_{\mathbf{a}}$, and is translation-invariant because

$$
\operatorname{Trans}_{\mathbf{u}}\left(\mathcal{L}_{\mathbf{a}}\right)(\mathbf{v}) \stackrel{\text { dfn }}{=} \mathcal{L}_{\mathbf{a}}(\mathbf{v}-\mathbf{u}) \stackrel{\text { dfn }}{=}\langle\overline{\mathbf{v}-\mathbf{u}}, \mathbf{a}\rangle=\mathcal{L}_{\mathbf{a}}(\mathbf{v})-\langle\overline{\mathbf{u}}, \mathbf{a}\rangle .
$$

Observe next that if $\mathcal{U}, \mathcal{U}^{\prime}: \mathbb{Z}_{6}^{I_{n}} \rightarrow \mathbb{Z}_{2}$ are both supported on $B_{n}$ then

$$
\begin{aligned}
& \mathcal{U}-\mathcal{U}^{\prime} \in D_{n}^{\perp} \quad \Leftrightarrow \quad\left\langle\left\langle\mathcal{U}, \mathbf{1}_{\mathbb{Z}_{6}^{I_{n}}}\right\rangle\right\rangle=\left\langle\left\langle\mathcal{U}^{\prime}, \mathbf{1}_{\mathbb{Z}_{6}^{I_{n}}}\right\rangle\right\rangle \\
& \text { and } \quad\left\langle\left\langle\mathcal{U}, \mathcal{L}_{\mathbf{a}}\right\rangle\right\rangle=\left\langle\left\langle\mathcal{U}^{\prime}, \mathcal{L}_{\mathbf{a}}\right\rangle\right\rangle \quad \forall \mathbf{a} \in C_{n} \\
& \Leftrightarrow \quad\left\langle\left\langle\mathcal{U}, \mathcal{L}_{\mathbf{1}_{I_{n}}}\right\rangle\right\rangle=\left\langle\left\langle\mathcal{U}^{\prime}, \mathcal{L}_{\mathbf{1}_{I_{n}}}\right\rangle\right\rangle \\
& \text { and }\left\langle\left\langle\mathcal{U}, \mathcal{L}_{\mathbf{a}}\right\rangle\right\rangle=\left\langle\left\langle\mathcal{U}^{\prime}, \mathcal{L}_{\mathbf{a}}\right\rangle\right\rangle \quad \forall \mathbf{a} \in C_{n} \\
& \text { (because spt } \mathcal{U}, \text { spt } \mathcal{U}^{\prime} \subseteq B_{n} \text { and }\left.\mathbf{1}_{\mathbb{Z}_{6}^{I_{n}}}\right|_{B_{n}}=\left.\mathcal{L}_{\mathbf{1}_{I_{n}}}\right|_{B_{n}} \text { ) } \\
& \Leftrightarrow \quad\left\langle\left\langle\mathcal{U}, \mathcal{L}_{\mathbf{a}}\right\rangle\right\rangle=\left\langle\left\langle\mathcal{U}^{\prime}, \mathcal{L}_{\mathbf{a}}\right\rangle\right\rangle \quad \forall \mathbf{a} \in C_{n} \\
& \text { (because we chose } C_{n} \text { to contain } \mathbf{1}_{I_{n}} \text { ) } \\
& \Leftrightarrow \quad \sum_{x \in I_{n}} \mathcal{U}\left(\mathbf{e}_{x}\right)\left\langle\overline{\mathbf{e}_{x}}, \mathbf{a}\right\rangle=\sum_{x \in I_{n}} \mathcal{U}^{\prime}\left(\mathbf{e}_{x}\right)\left\langle\overline{\mathbf{e}_{x}}, \mathbf{a}\right\rangle \quad \forall \mathbf{a} \in C_{n} \\
& \Leftrightarrow \quad \mathcal{U}-\left.\mathcal{U}^{\prime} \in \xi\left(C_{n}^{\perp}\right) \oplus \mathbf{0}\right|_{\mathbb{Z}_{6}^{I_{n}} \backslash B_{n}} .
\end{aligned}
$$

This proves the second part of the lemma.

Now, by the addition-invariance of $\mathrm{TS}_{\mathrm{Ham}}$ on $\mathbb{Z}_{2}^{\mathbb{Z}_{6}^{I_{n}}}$ (that is, invariance under addition of a fixed member of $\mathbb{Z}_{2}^{\mathbb{Z}_{6}^{I_{n}}}$, not under translation of the base $\mathbb{Z}_{6}^{I_{n}}$ ), to prove the third part of the lemma it suffices to show that for any $\mathcal{V}: \mathbb{Z}_{6}^{I_{n}} \rightarrow \mathbb{Z}_{2}$ with spt $\mathcal{V} \subseteq B_{n}$ the minimum

$$
\operatorname{TS}_{\mathrm{Ham} / D_{n}^{\perp}}\left(D_{n}^{\perp}, \mathcal{V}+D_{n}^{\perp}\right) \stackrel{\text { dfn }}{=} \min _{\mathcal{W} \in \mathcal{V}+D_{n}^{\perp}} \operatorname{TS}_{\mathrm{Ham}}(\mathbf{0}, \mathcal{W})
$$

is attained (possibly not uniquely) for some $\mathcal{W} \in \mathcal{V}+D_{n}^{\perp}$ that also has spt $\mathcal{W} \subseteq$ $B_{n}$. Adjusting $\mathcal{V}$ by some $B_{n}$-supported member of $D_{n}^{\perp}$ (thus, effectively, by a member of $\xi\left(C_{n}^{\perp}\right)$ ) if necessary, it further suffices to show that if $\mathcal{V}$ already minimizes $\operatorname{TS}_{\text {Ham }}(\mathbf{0}, \cdot)$ among members of its equivalence class modulo $D_{n}^{\perp} \cap\left(\mathbb{Z}_{2}^{B_{n}} \oplus\right.$ $\left.\left.\mathbf{0}\right|_{\mathbb{Z}_{6}^{I_{n}} \backslash B_{n}}\right)$, then any other $\mathcal{W}: \mathbb{Z}_{6}^{I_{n}} \rightarrow \mathbb{Z}_{2}$ with $\mathcal{V}-\mathcal{W} \in D_{n}^{\perp}$ has

$$
\operatorname{TS}_{\mathrm{Ham}}(\mathbf{0}, \mathcal{W}) \geq \operatorname{TS}_{\mathrm{Ham}}(\mathbf{0}, \mathcal{V})
$$

We will deduce this by showing how any $\mathcal{W}$ in $\mathcal{V}+D_{n}^{\perp}$ can be explicitly adjusted to another member of this equivalence class that is at least as close to $\mathbf{0}$ under $\mathrm{TS}_{\mathrm{Ham}}$ 
and is supported on $B_{n}$. Define the map $\mathcal{R}:\left.\mathbb{Z}_{2}^{\mathbb{Z}_{6}^{I_{n}}} \rightarrow \mathbb{Z}_{2}^{B_{n}} \oplus \mathbf{0}\right|_{\mathbb{Z}_{6}^{I_{n}} \backslash B_{n}}$ by

$$
\mathcal{R}(\mathcal{W})\left(\mathbf{e}_{x}\right):=\sum_{\mathbf{w} \in \mathbb{Z}_{6}^{I_{n}}} \mathcal{W}(\mathbf{w})\left\langle\overline{\mathbf{e}_{x}}, \overline{\mathbf{w}}\right\rangle:
$$

that is, $\mathcal{R}(\mathcal{W})$ is the $\mathbb{Z}_{2}$-valued indicator function of the subset of $B_{n}$ containing those basis vectors that appear with odd coefficients in the basis-decomposition of an odd number of the members of spt $\mathcal{W}$.

Now suppose that $\left(\mathbf{x}_{1}=\mathbf{0}, \mathbf{x}_{2}, \ldots, \mathbf{x}_{\ell+1}=\mathbf{0}\right)$ is a cycle in $\mathbb{Z}_{6}^{I_{n}}$ that covers spt $\mathcal{W}$. Since $d_{\text {Ham }}$ is a path metric on $\mathbb{Z}_{6}^{I_{n}}$ (corresponding to the usual nearest-neighbour graph on the Hamming cube over the one-dimensional space given by $\mathbb{Z}_{6}$ with its natural word metric), we may interpolate additional points along the shortest paths joining each pair $\left(\mathbf{x}_{i}, \mathbf{x}_{i+1}\right)$ and re-label so that the value

$$
\sum_{i=1}^{\ell} d_{\operatorname{Ham}}\left(\mathbf{x}_{i}, \mathbf{x}_{i+1}\right)
$$

in unchanged, but so that the consecutive points $\mathbf{x}_{i}, \mathbf{x}_{i+1}$ are now neighbours in this graph. Since the path starts and ends at $\mathbf{0}$, for any $x \in I_{n}$ and $\mathbf{w} \in \operatorname{spt} \mathcal{W}$ such that $w_{x} \neq 0$, the cycle must traverse some edge in direction $x$ (that is, move between two points of $\mathbb{Z}_{6}^{I_{n}}$ that differ by $\pm \mathbf{e}_{x}$ ) at least once before reaching $\mathbf{w}$, and at least once again on its way back to $\mathbf{0}$. It follows that the length of the cycle is at least twice the number of different basis vectors $\mathbf{e}_{i}$ that appear in the basis-representation of some $\mathbf{w} \in \mathcal{W}$, and this in turn is trivially bounded below by $|\operatorname{spt} \mathcal{R}(\mathcal{W})|$. On the other hand, the same reasoning easily shows that $\operatorname{TS}_{\text {Ham }}(\mathbf{0}, \mathcal{U})=2|\operatorname{spt} \mathcal{U}|+1$ whenever $\operatorname{spt} \mathcal{U} \subseteq B_{n}$ (since a cycle that starts at $\mathbf{0}$ and simply goes up to each member of $\operatorname{spt} \mathcal{U}$ in turn and then straight back down achieves the above lower bound). Therefore we certainly have

$$
\operatorname{TS}_{\text {Ham }}(\mathbf{0}, \mathcal{W}) \geq 2|\operatorname{spt} \mathcal{R}(\mathcal{W})|+1=\operatorname{TS}_{\text {Ham }}(\mathbf{0}, \mathcal{R}(\mathcal{W})) .
$$

It therefore suffices to show that $\mathcal{R}(\mathcal{W})-\mathcal{W} \in D_{n}^{\perp}$, since then the minimality of $\mathcal{V}$ among $B_{n}$-supported members of its equivalence class shows that

$$
\operatorname{TS}_{\text {Ham }}(\mathbf{0}, \mathcal{V}) \leq \operatorname{TS}_{\text {Ham }}(\mathbf{0}, \mathcal{R}(\mathcal{W}))
$$

so that concatenating this with the above inequality completes the proof.

Thus we must show that

$$
\left\langle\mathcal{W}, \eta+\mathcal{L}_{\mathbf{a}}\right\rangle=\left\langle\mathcal{R}(\mathcal{W}), \eta+\mathcal{L}_{\mathbf{a}}\right\rangle
$$


for all $\eta \in \mathbb{Z}_{2}$ and $\mathbf{a} \in C_{n}$; in fact we will go slightly further and show that this holds for all $\mathbf{a} \in \mathbb{Z}_{2}^{B_{n}}$. By the $\mathbb{Z}_{2}$-linearity of the map $\mathbf{a} \mapsto \mathcal{L}_{\mathbf{a}}$ it suffices to prove separately that

$$
\left\langle\left\langle\mathcal{W}, \mathbf{1}_{\mathbb{Z}_{6}^{I_{n}}}\right\rangle\right\rangle=\left\langle\left\langle\mathcal{R}(\mathcal{W}), \mathbf{1}_{\mathbb{Z}_{6}^{I_{n}}}\right\rangle\right\rangle, \quad \text { i.e. } \quad|\operatorname{spt} \mathcal{W}| \equiv|\operatorname{spt} \mathcal{R}(\mathcal{W})| \bmod 2,
$$

and that

$$
\left\langle\left\langle\mathcal{W}, \mathcal{L}_{\overline{\mathbf{e}_{x}}}\right\rangle\right\rangle=\left\langle\left\langle\mathcal{R}(\mathcal{W}), \mathcal{L}_{\overline{\mathbf{e}_{x}}}\right\rangle\right\rangle \quad \forall x \in I_{n}
$$

To prove the first of these equalities, observe that

$$
\begin{aligned}
|\operatorname{spt} \mathcal{R}(\mathcal{W})| & =\mid\left\{x \in I_{n}: \mathbf{e}_{x}\right. \text { appears with odd coeff. } \\
& \text { in an odd number of } \mathbf{w} \in \operatorname{spt} \mathcal{W}\} \mid \\
& \equiv \sum_{x \in I_{n}}\left|\left\{\mathbf{w} \in \operatorname{spt} \mathcal{W}:\left\langle\overline{\mathbf{e}}_{x}, \overline{\mathbf{w}}\right\rangle=1\right\}\right| \bmod 2 \\
& \equiv \sum_{x \in I_{n}} \sum_{\mathbf{w} \in \operatorname{spt} \mathcal{W}}\left\langle\overline{\mathbf{e}_{x}}, \overline{\mathbf{w}}\right\rangle \bmod 2 \\
& \equiv \sum_{\mathbf{w} \in \mathbb{Z}_{6}^{I_{n}}} \mathcal{W}(\mathbf{w})\left\langle\overline{\mathbf{w}}, \sum_{x \in I_{n}} \overline{\mathbf{e}_{x}}\right\rangle \bmod 2 \\
& \equiv\left\langle\left\langle\mathcal{W}, \mathcal{L}_{\mathbf{1}_{I_{n}}}\right\rangle\right\rangle \bmod 2 .
\end{aligned}
$$

Since by construction $\mathbf{1}_{I_{n}} \in C_{n}$ and so $\mathcal{L}_{\mathbf{1}_{I_{n}}} \in D_{n}$, and also $\mathbf{1}_{\mathbb{Z}_{6}^{I_{n}}} \in D_{n}$, and we know that $\mathcal{W}-\mathcal{V} \in D_{n}^{\perp}$, we have

$$
\begin{aligned}
\left\langle\left\langle\mathcal{W}, \mathcal{L}_{1_{I_{n}}}\right\rangle\right\rangle & =\left\langle\left\langle\mathcal{V}, \mathcal{L}_{1_{I_{n}}}\right\rangle\right\rangle \\
& \equiv|\mathcal{V}| \bmod 2 \\
& \left.\equiv\left\langle\mathcal{V}, \mathbf{1}_{\mathbb{Z}_{6}^{I_{n}}}\right\rangle\right\rangle \\
& =\left\langle\left\langle\mathcal{W}, \mathbf{1}_{\mathbb{Z}_{6}^{I_{n}}}\right\rangle\right\rangle \\
& \equiv|\operatorname{spt} \mathcal{W}| \bmod 2,
\end{aligned}
$$

so concatenating these equations gives the result.

To prove the second equality, we simply observe that

$$
\begin{array}{r}
\left\langle\left\langle\mathcal{W}, \mathcal{L}_{\overline{\mathbf{e}_{x}}}\right\rangle=\sum_{\mathbf{w}} \mathcal{W}(\mathbf{w})\left\langle\overline{\mathbf{w}}, \overline{\mathbf{e}_{x}}\right\rangle=\left\langle\sum_{\mathbf{w}} \sum_{y \in I_{n}} \mathcal{W}(\mathbf{w})\left\langle\overline{\mathbf{e}_{y}}, \overline{\mathbf{w}}\right\rangle \overline{\mathbf{e}_{y}}, \overline{\mathbf{e}_{x}}\right\rangle\right. \\
=\sum_{\mathbf{w}} \mathcal{R}(\mathcal{W})(\mathbf{w})\left\langle\overline{\mathbf{e}_{x}}, \overline{\mathbf{w}}\right\rangle=\left\langle\left\langle\mathcal{R}(\mathcal{W}), \mathcal{L}_{\left.\overline{\mathbf{e}_{x}}\right\rangle}\right\rangle,\right.
\end{array}
$$

as required. 
Corollary 4.3. The map

$$
\kappa_{n}^{\circ}: \mathbb{Z}_{2}^{I_{n}} \rightarrow \mathbb{Z}_{2}^{\mathbb{Z}_{6}^{I_{n}}} / D_{n}^{\perp}: \mathbf{a} \mapsto \mathbf{1}_{\left\{\mathbf{e}_{x} \in B_{n}:\left\langle\overline{\mathbf{e}}_{x}, \mathbf{a}\right\rangle=1\right\}}+D_{n}^{\perp}
$$

has kernel precisely $C_{n}^{\perp}$, and the resulting quotient map

$$
\kappa_{n}: \mathbb{Z}_{2}^{I_{n}} / C_{n}^{\perp} \hookrightarrow \mathbb{Z}_{2}^{\mathbb{Z}_{6}^{I_{n}}} / D_{n}^{\perp}
$$

is a bi-Lipschitz embedding of $d_{\mathrm{Ham} / C_{n}^{\perp}}$ into $\mathrm{TS}_{\mathrm{Ham} / D_{n}^{\perp}}$ with distortion at most 2 and expansion ratio at most 2 .

Proof The identification of the kernel of $\kappa_{n}^{\circ}$ is already contained in the second part of Lemma 4.2. so we need only prove the bi-Lipschitz and expansion ratio bounds.

To this end, suppose that $\mathbf{a}, \mathbf{b} \in \mathbb{Z}_{2}^{I_{n}}$. Then the images $\kappa_{n}\left(\mathbf{a}+C_{n}^{\perp}\right)$ and $\kappa_{n}\left(\mathbf{b}+C_{n}^{\perp}\right)$ are represented modulo $D_{n}^{\perp}$ by the functions $\mathcal{A}:=\mathbf{1}_{\left\{\mathbf{e}_{x} \in B_{n}:\left\langle\overline{\mathbf{e}}_{x}, \mathbf{a}\right\rangle=1\right\}}$ and $\mathcal{B}:=$ $\mathbf{1}_{\left\{\mathbf{e}_{x} \in B_{n}:\left\langle\overline{\mathbf{e}}_{x}, \mathbf{b}\right\rangle=1\right\}}$. By the third part of Lemma 4.2 the distance

$$
\mathrm{TS}_{\mathrm{Ham} / D_{n}^{\perp}}\left(\mathcal{A}+D_{n}^{\perp}, \mathcal{B}+D_{n}^{\perp}\right)
$$

is attained as $\operatorname{TS}_{\mathrm{Ham}}\left(\mathcal{A}^{\prime}, \mathcal{B}^{\prime}\right)$ for some $\mathcal{A}^{\prime} \in \mathcal{A}+\left(\left.\xi\left(C_{n}^{\perp}\right) \oplus \mathbf{0}\right|_{\mathbb{Z}_{6}^{I_{n}} \backslash B_{n}}\right)$ and $\mathcal{B}^{\prime} \in$ $\mathcal{B}+\left(\left.\xi\left(C_{n}^{\perp}\right) \oplus \mathbf{0}\right|_{\mathbb{Z}_{6}^{I_{n}} \backslash B_{n}}\right)$, and given these it is simply equal to $2\left|\operatorname{spt}\left(\mathcal{A}^{\prime}+\mathcal{B}^{\prime}\right)\right|+1$. However, we may clearly represent $\mathcal{A}^{\prime}+D_{n}^{\perp}=\kappa_{n}^{\circ}\left(\mathbf{a}^{\prime}\right)$ and $\mathcal{B}^{\prime}+D_{n}^{\perp}=\kappa_{n}^{\circ}\left(\mathbf{b}^{\prime}\right)$, and hence deduce from the first part of the corollary that $\mathbf{a}^{\prime} \in \mathbf{a}+C_{n}^{\perp}$ and $\mathbf{b}^{\prime} \in \mathbf{b}+C_{n}^{\perp}$. It follows that $\operatorname{TS}_{\mathrm{Ham}}\left(\mathcal{A}^{\prime}, \mathcal{B}^{\prime}\right)$ is precisely $2 d_{\mathrm{Ham}}\left(\mathbf{a}^{\prime}, \mathbf{b}^{\prime}\right)+1$, and thus that

$$
\mathrm{TS}_{\mathrm{Ham} / D_{n}^{\perp}}\left(\kappa_{n}\left(\mathbf{a}+C_{n}^{\perp}\right), \kappa_{n}\left(\mathbf{b}+C_{n}^{\perp}\right)\right) \geq 2 d_{\mathrm{Ham} / C_{n}^{\perp}}\left(\mathbf{a}+C_{n}^{\perp}, \mathbf{b}+C_{n}^{\perp}\right)+1 .
$$

The reverse inequality also follows simply because for any $\mathbf{a}^{\prime} \in \mathbf{a}+C_{n}^{\perp}$ and $\mathbf{b}^{\prime} \in \mathbf{b}+C_{n}^{\perp}$ the images $\kappa_{n}^{\circ}\left(\mathbf{a}^{\prime}\right)$ and $\kappa_{n}^{\circ}\left(\mathbf{b}^{\prime}\right)$ are candidates for being set equal to $\mathcal{A}^{\prime}$ and $\mathcal{B}^{\prime}$ above.

Thus in fact

$$
\mathrm{TS}_{\mathrm{Ham} / D_{n}^{\perp}}\left(\kappa_{n}\left(\mathbf{a}+C_{n}^{\perp}\right), \kappa_{n}\left(\mathbf{b}+C_{n}^{\perp}\right)\right)=2 d_{\mathrm{Ham} / C_{n}^{\perp}}\left(\mathbf{a}+C_{n}^{\perp}, \mathbf{b}+C_{n}^{\perp}\right)+1,
$$

and since all distances involved are at least 1 this gives rise to the asserted bounds on bi-Lipschitz constant and expansion ratio. 


\subsection{Completion of the proof}

We will now show how the quotients of finite-dimensional product spaces studied in the preceding subsection can be simultaneously bi-Lipschitzly recovered from a single translation-invariant quotient of the metric $\rho_{\left(\left(\mathbb{Z}_{2}^{\oplus\left(\mathbb{Z}_{6} \zeta G\right)}\right) \rtimes\left(\mathbb{Z}_{6} \imath G\right)\right)_{0}}$ on $\left(\left(\mathbb{Z}_{2}^{\oplus\left(\mathbb{Z}_{6} \imath G\right)}\right) \rtimes\left(\mathbb{Z}_{6} \curlywedge G\right)\right)_{0}=\mathbb{Z}_{2}^{\oplus\left(\mathbb{Z}_{6} \imath G\right)}$.

The tricky part is that we must find a single subspace $V \leq \mathbb{Z}_{2}^{\oplus\left(\mathbb{Z}_{6} \imath G\right)}$ such that quotienting by it mimics the finite-dimensional quotienting by $D_{n}^{\perp}$ studied above at each of an increasing sequence of 'scales', indexed by $n$, so that these scales do not 'interact'. This is crucial to our recovery of a copy of $\left(\mathbb{Z}_{2}^{\mathbb{Z}_{6}^{I_{n}}} / D_{n}^{\perp}, \mathrm{TS}_{\mathrm{Ham} / D_{n}^{\perp}}\right)$ (and hence of $\left(\mathbb{Z}_{2}^{I_{n}} / C_{n}^{\perp}, d_{\mathrm{Ham} / C_{n}^{\perp}}\right)$ by Corollary 4.3) for each $n$. It is here that we will see most clearly the usefulness of making a second extension to construct our overall group.

Recall that given the group $G$ and its metric $\rho$, we can pick positive reals $r_{n}$, subsets $I_{n}$ and points $y_{n}$ as in the preceding section. Clearly by passing to a subsequence if necessary, we may always assume that the sequence

$$
r_{1}<\rho\left(y_{1}, e_{G}\right)<r_{2}<\rho\left(y_{2}, e_{G}\right)<\ldots
$$

grows as fast as we please. We will henceforth refer to this as the sequence of scales.

We will now introduce the feature of $\mathbb{Z}_{6}$ (as opposed, say, to $\mathbb{Z}_{4}$ ) that makes it suitable for our construction: if 1 is its generator, and if we let $\mathcal{A}$ be the $\mathbb{Z}_{2}$-valued indicator function of the subset $\{0,1,3,4\}$, then $\mathcal{A}$ has the property (as may easily be checked) that it and its distinct translates $\mathcal{A}(\cdot-v)$ for $v \in \mathbb{Z}_{6}$ (of which there are only three, since $\mathcal{A}(\cdot-3)=\mathcal{A}$ ) have no linear combination modulo 2 that is equal to the indicator function $\mathbf{1}_{\mathbb{Z}_{6}}$. Let us now fix this indicator function $\mathcal{A}$ for the rest of this paper.

Define the sequence of maps

$$
\mathcal{Q}_{n}: \mathbb{Z}_{6}^{\oplus\left(G \backslash\left(I_{1} \cup I_{2} \cup \cdots \cup I_{n} \cup\left\{y_{1}, y_{2}, \ldots, y_{n}\right\}\right)\right)} \times \mathbb{Z}_{2}^{\mathbb{Z}_{6}^{I_{n}}} \times \mathbb{Z}_{6} \rightarrow \mathbb{Z}_{2}^{\oplus \mathbb{Z}_{6}^{\oplus G}}
$$

by setting

$\mathcal{Q}_{n}(\mathbf{u}, \mathcal{W}, u): \mathbf{w} \mapsto \delta_{\mathbf{u}}\left(\left.\mathbf{w}\right|_{G \backslash\left(I_{1} \cup I_{2} \cup \cdots \cup I_{n} \cup\left\{y_{1}, y_{2}, \ldots, y_{n}\right\}\right)}\right) \cdot \mathcal{W}\left(\left.\mathbf{w}\right|_{I_{n}}\right) \cdot \mathcal{A}\left(w_{y_{n}}-u\right)$, and define also

$$
\tilde{\mathcal{Q}}_{n}: \mathbb{Z}_{6}^{\oplus\left(G \backslash\left(I_{1} \cup I_{2} \cup \cdots \cup I_{n} \cup\left\{y_{1}, y_{2}, \ldots, y_{n}\right\}\right)\right)} \times \mathbb{Z}_{2}^{\mathbb{Z}_{6}^{I_{n}}} \times \mathbb{Z}_{6} \rightarrow \mathbb{Z}_{2}^{\oplus\left(\mathbb{Z}_{6} \imath G\right)}
$$


by

$$
\tilde{\mathcal{Q}}_{n}(\mathbf{u}, \mathcal{W}, u)(\mathbf{w}, g)= \begin{cases}\mathcal{Q}_{n}(\mathbf{u}, \mathcal{W}, u)(\mathbf{w}) & \text { if } g=e_{G} \\ 0 & \text { else. }\end{cases}
$$

Thus $\mathcal{Q}_{n}(\mathbf{u}, \mathcal{W}, u)$ is the $\mathbb{Z}_{2}$-valued indicator function of the set of those $\mathbf{w} \in \mathbb{Z}_{6}^{\oplus G}$ such that

- the restriction of $\mathbf{w}$ to the indices in $G \backslash\left(I_{1} \cup I_{2} \cup \cdots \cup I_{n} \cup\left\{y_{1}, y_{2}, \ldots, y_{n}\right\}\right)$ agrees with $\mathbf{u}$,

- the restriction $\left.\mathbf{w}\right|_{I_{n}}$ is a member of $\operatorname{spt} \mathcal{W}$, and

- the single coordinate $w_{y_{n}}\left(=\left.\mathbf{w}\right|_{\left\{y_{n}\right\}}\right)$ is in the translated set $\operatorname{Trans}_{u}(\operatorname{spt} \mathcal{A})$.

These maps $\mathcal{Q}_{n}$ and $\tilde{\mathcal{Q}}_{n}$ will both underpin the construction of $V$ and form the building blocks of our final embeddings into $\mathbb{Z}_{2}^{\oplus\left(\mathbb{Z}_{6} \ell G\right)}$. The slightly mysterious inclusion of a copy of $\mathcal{A}$ in this definition will be important for keeping track of certain possible cancelations when we add translated copies of images under these maps $\mathcal{Q}_{n}$, and will be clarified shortly.

Some of the immediate properties of $\mathcal{Q}_{n}$ seem worth making explicit:

Definition 4.4 (Linearity; translation-covariance). Each map $\mathcal{Q}_{n}$ is linear in its second argument:

$$
\mathcal{Q}_{n}(\mathbf{u}, \mathcal{W}, u)+\mathcal{Q}_{n}\left(\mathbf{u}, \mathcal{W}^{\prime}, u\right)=\mathcal{Q}_{n}\left(\mathbf{u}, \mathcal{W}+\mathcal{W}^{\prime}, u\right) \quad \forall \mathcal{W}, \mathcal{W}^{\prime} \in \mathbb{Z}_{2}^{\mathbb{Z}_{6}^{I_{n}}}
$$

for fixed $\mathbf{u}$ and $u$. We will refer to this as the linearity property of $\mathcal{Q}_{n}$.

Each map $\mathcal{Q}_{n}$ also behaves well under translations in all three arguments:

$$
\begin{aligned}
& \operatorname{Trans}_{\mathbf{w}} \mathcal{Q}_{n}(\mathbf{u}, \mathcal{W}, u)(\cdot) \\
&=\mathcal{Q}_{n}\left(\mathbf{u}+\left.\mathbf{w}\right|_{G \backslash\left(I_{1} \cup \cdots \cup I_{n} \cup\left\{y_{1}, \ldots, y_{n}\right\}\right)}, \operatorname{Trans}_{\left.\mathbf{w}\right|_{I_{n}}} \mathcal{W}, u+w_{y_{n}}\right)(\cdot) \\
& \forall \mathbf{w}, \mathbf{u}, \mathcal{W}, u .
\end{aligned}
$$

We will refer to this as the translation-covariance property of $\mathcal{Q}_{n}$.

Having made these definitions, we can state the last main result on the route to Theorem 3.3 . 
Proposition 4.5. If $\left(D_{n}\right)_{n \geq 1}$ is as in Lemma 4.2 and the sequence of scales grows sufficiently fast then there is a $\mathbb{Z}_{2}$-subspace $V \leq \mathbb{Z}_{2}^{\oplus\left(\mathbb{Z}_{6} \imath G\right)}$ that is invariant under the coordinate right-translation action of $\mathbb{Z}_{6} 2 G$ and for which the following holds: if for each $n \geq 1$ we define the mapping

$$
\psi_{n}^{\circ}: \mathbb{Z}_{2}^{\mathbb{Z}_{6}^{I_{n}}} \rightarrow \mathbb{Z}_{2}^{\oplus\left(\mathbb{Z}_{6} \imath G\right)} / V: \mathcal{W} \mapsto \tilde{\mathcal{Q}}_{n}\left(\mathbf{0}_{G \backslash\left(I_{1} \cup \cdots \cup I_{n} \cup\left\{y_{1}, \ldots, y_{n}\right\}\right)}, \mathcal{W}, 0\right)+V,
$$

then

1. $\psi_{n}^{\circ}$ is $\mathbb{Z}_{2}$-linear with kernel $D_{n}^{\perp}$;

2. the resulting quotient map

$$
\psi_{n}: \mathbb{Z}_{2}^{\mathbb{Z}_{6}^{I_{n}}} / D_{n}^{\perp} \hookrightarrow \mathbb{Z}_{2}^{\oplus\left(\mathbb{Z}_{6} \imath G\right)} / V \stackrel{\text { inclusion }}{\hookrightarrow}\left(\mathbb{Z}_{2}^{\oplus\left(\mathbb{Z}_{6} \imath G\right)} / V\right) \rtimes\left(\mathbb{Z}_{6} \curlywedge G\right)
$$

is a bi-Lipschitz embedding of $\mathrm{TS}_{\mathrm{Ham} / D_{n}^{\perp}}$ into $\rho_{\left(\mathbb{Z}_{2}^{\oplus\left(\mathbb{Z}_{6} \imath G\right)} / V\right) \rtimes\left(\mathbb{Z}_{6} \imath G\right)}$ with distortion at most $\mathrm{O}(M)$ and expansion ratio $\mathrm{O}\left(r_{n}\right)$.

We will prove this proposition in several steps in this subsection, but let us first see how it and Corollary 4.3 together imply Theorem 3.3 and so complete the proof of Theorem 1.1

Proof of Theorem 3.3 from Proposition 4.5 This follows simply by setting

$$
\varphi_{n}:=\psi_{n} \circ \kappa_{n}: \mathbb{Z}_{2}^{I_{n}} / C_{n}^{\perp} \hookrightarrow\left(\mathbb{Z}_{2}^{\oplus\left(\mathbb{Z}_{6} \imath G\right)} / V\right) \rtimes\left(\mathbb{Z}_{6} \curlywedge G\right),
$$

since the estimates of Corollary 4.3 and Proposition 4.5 show that $\varphi_{n}$ has distortion at most $2 \cdot(4 M)=8 M$, which does not depend on $n$, and expansion ratio $\mathrm{O}\left(r_{n}\right)$, where the dimension of the cube $\mathbb{Z}_{2}^{I_{n}}$ is $d_{n} \geq 10^{r_{n}}$ and so the diameter of its quotient $\mathbb{Z}_{2}^{I_{n}} / C_{n}^{\perp}$, which has $L_{p}$-distortion proportional to this diameter, is also $\Omega\left(d_{n}\right)$, which grows faster than any power of $r_{n}$.

We will construct the subspace $V$ that we need in a number of steps. First, given the subspaces $D_{n} \leq \mathbb{Z}_{2}^{\mathbb{Z}_{6}^{I_{n}}}$ obtained in Lemma 4.2, let

$U_{n}:=\operatorname{span}_{\mathbb{Z}_{2}}\left\{\mathcal{Q}_{n}(\mathbf{u}, \mathcal{W}, u): \mathbf{u} \in \mathbb{Z}_{6}^{\oplus\left(G \backslash\left(I_{1} \cup \cdots \cup I_{n} \cup\left\{y_{1}, \ldots, y_{n}\right\}\right)\right)}, \mathcal{W} \in D_{n}^{\perp}, u \in \mathbb{Z}_{6}\right\}$.

Let us also define

$U_{n}^{+}:=\operatorname{span}_{\mathbb{Z}_{2}}\left\{\mathcal{Q}_{n}(\mathbf{u}, \mathcal{W}, u): \mathbf{u} \in \mathbb{Z}_{6}^{\oplus\left(G \backslash\left(I_{1} \cup \cdots \cup I_{n} \cup\left\{y_{1}, \ldots, y_{n}\right\}\right)\right)}, \mathcal{W} \in \mathbb{Z}_{2}^{\mathbb{Z}_{6}^{I_{n}}}, u \in \mathbb{Z}_{6}\right\}$, 
so that $U_{n} \leq U_{n}^{+}$.

It follows at once from the translation-covariance of $Q_{n}$ and the translation-invariance

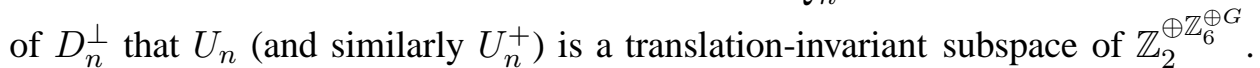
Now we let $U:=\sum_{n \geq 1} U_{n}$, so that this is still a translation-invariant $\mathbb{Z}_{2}$-subspace

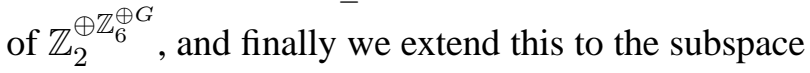

$$
\begin{array}{r}
V:=\left\{\mathcal{V} \in \mathbb{Z}_{2}^{\oplus\left(\mathbb{Z}_{6} \zeta G\right)}: \text { the map }\left(w_{g}\right)_{g \in G} \mapsto \mathcal{V}\left(\left(w_{g g_{1}^{-1}}\right)_{g \in G}, g_{1}\right)\right. \\
\text { lies in } \left.U \text { for every } g_{1} \in G\right\}
\end{array}
$$

of $\mathbb{Z}_{2}^{\oplus\left(\mathbb{Z}_{6} \imath G\right)}$, which is manifestly invariant under all right-translations by members of $\mathbb{Z}_{6} \succ G$. This is the subspace that we will show enjoys the properties listed in Proposition 4.5

Our first step is the following simple strengthening of Lemma 2.6, which will reduce our proof of Proposition 4.5 to a study of a simpler map into the space $\mathbb{Z}_{2}^{\oplus\left(\mathbb{Z}_{6} \imath G\right)_{0}} / U$.

Lemma 4.6. The composition of mappings

$$
\begin{array}{llll}
\mathbb{Z}_{2}^{\oplus\left(\mathbb{Z}_{6} \zeta G\right)_{0}} / U & \hookrightarrow \mathbb{Z}_{2}^{\oplus\left(\mathbb{Z}_{6} \imath G\right)} / V & \hookrightarrow\left(\mathbb{Z}_{2}^{\oplus\left(\mathbb{Z}_{6} \zeta G\right)} / V\right) \rtimes\left(\mathbb{Z}_{6} \curlywedge G\right) \\
\mathcal{U}+U & \mapsto\left(\left.\mathcal{U} \oplus \mathbf{0}\right|_{\left(\mathbb{Z}_{6} \zeta G\right) \backslash\left(\mathbb{Z}_{6} \zeta G\right)_{0}}\right)+V & \mapsto\left(\left(\left.\mathcal{U} \oplus \mathbf{0}\right|_{\left(\mathbb{Z}_{6} \zeta G\right) \backslash\left(\mathbb{Z}_{6} \zeta G\right)_{0}}\right)+V,\left(\mathbf{0}, e_{G}\right)\right)
\end{array}
$$

is a well-defined $\mathbb{Z}_{2}$-linear injection, and moreover is a 2-bi-Lipschitz embedding of the metric $\operatorname{TS}_{\rho_{\left(\mathbb{Z}_{6} \imath G\right)_{0}},\left(\mathbf{0}, e_{G}\right) / U}$ on $\mathbb{Z}_{2}^{\oplus\left(\mathbb{Z}_{6} \imath G\right)_{0}} / U$ into $\rho_{\left(\mathbb{Z}_{2}^{\oplus\left(\mathbb{Z}_{6} \imath G\right)} / V\right) \rtimes\left(\mathbb{Z}_{6} \imath G\right)}$ with expansion ratio $\Theta(1)$.

Proof We have already seen in Lemma 2.6 that the second part of our composition,

$$
\begin{array}{ll}
\mathbb{Z}_{2}^{\oplus\left(\mathbb{Z}_{6} \curlywedge G\right)} / V & \hookrightarrow\left(\mathbb{Z}_{2}^{\oplus\left(\mathbb{Z}_{6} \curlywedge G\right)} / V\right) \rtimes\left(\mathbb{Z}_{6} \succ G\right) \\
\mathcal{V}+V & \mapsto\left(\mathcal{V}+V,\left(\mathbf{0}, e_{G}\right)\right)
\end{array}
$$

is a 2-bi-Lipschitz embedding of $\operatorname{TS}_{\rho_{\left(\mathbb{Z}_{6} \curlywedge G\right)},\left(\mathbf{0}, e_{G}\right) / V}$ into $\rho_{\left(\mathbb{Z}_{2}^{\oplus\left(\mathbb{Z}_{6} \imath G\right)} / V\right) \rtimes\left(\mathbb{Z}_{6} \imath G\right)}$ with expansion ratio $\Theta(1)$; therefore it will suffice to show that the first part,

$$
\mathbb{Z}_{2}^{\oplus\left(\mathbb{Z}_{6} \imath G\right)_{0}} / U \hookrightarrow \mathbb{Z}_{2}^{\oplus\left(\mathbb{Z}_{6} \imath G\right)} / V
$$

is a well-defined $\mathbb{Z}_{2}$-linear injection that is an isometric embedding of $\operatorname{TS}_{\rho_{\left(\mathbb{Z}_{6} \ell G\right)},},\left(\mathbf{0}, e_{G}\right) / U$ into $\operatorname{TS}_{\rho_{\left(\mathbb{Z}_{6} \zeta G\right)},\left(\mathbf{0}, e_{G}\right) / V}$.

Linearity is immediate, and the correctness of the definition and injectivity hold because from the definition of $V$ we see that if $\mathcal{U}, \mathcal{U}^{\prime} \in \mathbb{Z}_{2}^{\oplus\left(\mathbb{Z}_{6} \zeta G\right)}$ are both supported 
on $\left(\mathbb{Z}_{6} \prec G\right)_{0}$, then they differ by a member of $V$ if and only if $\left.\mathcal{U}\right|_{\left(\mathbb{Z}_{6} \prec G\right)_{0}}$ and $\left.\mathcal{U}^{\prime}\right|_{\left(\mathbb{Z}_{6} \zeta G\right)_{0}}$ differ by a member of $U$.

Isometricity follows from some simple consideration of the definition of the pinned traveling salesman metric. Let us identify $\mathbb{Z}_{2}$-valued functions on $\left(\mathbb{Z}_{6} \succ G\right)_{0}$ with their extensions by 0 to $\mathbb{Z}_{6} \zeta G$ to lighten notation. Given any $\mathcal{W} \in V$, let $\mathcal{W}_{0}$ be the function $\mathcal{W} \cdot \mathbf{1}_{\left(\mathbb{Z}_{6} \imath G\right)_{0}}$ obtained by replacing $\mathcal{W}$ with the identically-zero function outside the zero section $\left(\mathbb{Z}_{6} \zeta G\right)_{0}$, and observe from the definition of $V$ that $\mathcal{W}_{0} \in$ $V$ also. Now if $\mathcal{U}$ and $\mathcal{U}^{\prime}$ are supported on the zero section, it follows that $\operatorname{spt}(\mathcal{U}-$ $\left.\mathcal{U}^{\prime}+\mathcal{W}\right) \supseteq \operatorname{spt}\left(\mathcal{U}-\mathcal{U}^{\prime}+\mathcal{W}_{0}\right)$, and now appealing to the monotonicity property of Lemma2.5 we deduce that for such $\mathcal{U}$ and $\mathcal{U}^{\prime}$ the quotient-metric distance

$$
\operatorname{TS}_{\rho_{\left(\mathbb{Z}_{6} \imath G\right)},\left(\mathbf{0}, e_{G}\right) / V}\left(\mathcal{U}+V, \mathcal{U}^{\prime}+V\right)
$$

is attained as $\operatorname{TS}_{\rho_{\left(\mathbb{Z}_{6} \zeta G\right)},\left(\mathbf{0}, e_{G}\right)}\left(\mathcal{U}, \mathcal{U}^{\prime}+\mathcal{W}\right)$ for some $\mathcal{W}$ also supported on $\left(\mathbb{Z}_{6} \succ G\right)_{0}$, and hence agrees with

$$
\operatorname{TS}_{\rho_{\left(\mathbb{Z}_{6} \imath G\right)_{0}},\left(\mathbf{0}, e_{G}\right) / U}\left(\mathcal{U}+U, \mathcal{U}^{\prime}+U\right),
$$

as required.

We next prove the two lemmas that will underpin the main estimates involved in the proof of Proposition 4.5

Lemma 4.7. Suppose that $\eta \in \mathbb{Z}_{2}, m \geq 1$ is an integer and that we are given

- a fixed point $\mathbf{u}^{\circ} \in \mathbb{Z}_{6}^{\oplus\left(G \backslash\left(I_{1} \cup \cdots \cup I_{m} \cup\left\{y_{1}, \ldots, y_{m}\right\}\right)\right)}$, and

- for each $n \leq m$, a finite family of functions

$$
\mathcal{F}_{n}:=\left\{\mathcal{Q}_{n}\left(\mathbf{u}_{1}^{(n)}, \mathcal{W}_{1}^{(n)}, u_{1}^{(n)}\right), \mathcal{Q}_{n}\left(\mathbf{u}_{2}^{(n)}, \mathcal{W}_{2}^{(n)}, u_{2}^{(n)}\right), \ldots, \mathcal{Q}_{n}\left(\mathbf{u}_{i_{n}}^{(n)}, \mathcal{W}_{i_{n}}^{(n)}, u_{i_{n}}^{(n)}\right)\right\}
$$

from $U_{n}^{+}$such that $\left.\mathbf{u}_{i}^{(n)}\right|_{\left(G \backslash\left(I_{1} \cup \cdots \cup I_{m} \cup\left\{y_{1}, \ldots, y_{m}\right\}\right)\right)}=\mathbf{u}^{\circ}$ for all $i \leq i_{n}$

such that

$$
\sum_{n=1}^{m} \sum_{i=1}^{i_{n}} \mathcal{Q}_{n}\left(\mathbf{u}_{i}^{(n)}, \mathcal{W}_{i}^{(n)}, u_{i}^{(n)}\right)=\eta \mathbf{1}_{\left\{\mathbf{u}^{\circ}\right\} \times \mathbb{Z}_{6}^{I_{1} \cup \cdots \cup I_{m} \cup\left\{y_{1}, \ldots, y_{m}\right\}} .} .
$$

Then we must have $\eta=0$ and

$$
\sum_{i=1}^{i_{n}} \mathcal{Q}_{n}\left(\mathbf{u}_{i}^{(n)}, \mathcal{W}_{i}^{(n)}, u_{i}^{(n)}\right)=0
$$

for each $n \leq m$ separately. 
Remark It will be important that we have this lemma available for basis members of the larger spaces $U_{n}^{+}$, not just of $U_{n}$.

Proof This will follow by induction on the number among the families $\mathcal{F}_{1}, \mathcal{F}_{2}$, $\ldots, \mathcal{F}_{m}$ that are nonempty.

Base clause Suppose that only one $\mathcal{F}_{n}$ is nonempty, in which case our assumption reads

$$
\sum_{i=1}^{i_{n}} \mathcal{Q}_{n}\left(\mathbf{u}_{i}^{(n)}, \mathcal{W}_{i}^{(n)}, u_{i}^{(n)}\right)=\eta \mathbf{1}_{\left\{\mathbf{u}^{\circ}\right\} \times \mathbb{Z}_{6}^{I_{1} \cup \cdots \cup I_{m} \cup\left\{y_{1}, \ldots, y_{m}\right\}}},
$$

and we need only show that $\eta=0$. We prove this by contradiction, so suppose instead that $\eta=1$. The function $\mathcal{Q}_{n}\left(\mathbf{u}_{i}^{(n)}, \mathcal{W}_{i}^{(n)}, u_{i}^{(n)}\right)$ is supported on the (infinitedimensional) cylinder $\left\{\mathbf{u}_{i}^{(n)}\right\} \times \mathbb{Z}_{6}^{\left(I_{1} \cup \cdots \cup I_{n} \cup\left\{y_{1}, \ldots, y_{n}\right\}\right)}$, and so if $\mathbf{u}_{i}^{(n)} \neq \mathbf{u}_{j}^{(n)}$ then $\mathcal{Q}_{n}\left(\mathbf{u}_{i}^{(n)}, \mathcal{W}_{i}^{(n)}, u_{i}^{(n)}\right)$ and $\mathcal{Q}_{n}\left(\mathbf{u}_{j}^{(n)}, \mathcal{W}_{j}^{(n)}, u_{j}^{(n)}\right)$ are disjointly supported. Now suppose that $\mathbf{v}$ is a vector that appears in the list $\mathbf{u}_{1}^{(n)}, \ldots, \mathbf{u}_{i_{n}}^{(n)}$ (so that by assumption, $\mathbf{v}$ is an extension of $\mathbf{u}^{\circ}$ ), and let $j_{1}<j_{2}<\ldots<j_{k}$ be those values of $i \leq i_{n}$ where it appears. It follows from the above equation that

$\sum_{s=1}^{k} \mathcal{Q}_{n}\left(\mathbf{v}, \mathcal{W}_{j_{s}}^{(n)}, u_{j_{s}}^{(n)}\right)=1 \quad$ on $\{\mathbf{v}\} \times \mathbb{Z}_{6}^{I_{n} \cup\left\{y_{n}\right\}} \times\left\{\mathbf{0}_{I_{1} \cup \ldots \cup I_{n-1} \cup\left\{y_{1}, \ldots, y_{n-1}\right\}}\right\}$.

Now pick any $\mathbf{w} \in \mathbb{Z}_{6}^{I_{n}}$; the above equation requires that $\mathcal{W}_{j_{s}}^{(n)}(\mathbf{w})=1$ for at least one $s \leq k$. Letting $s_{1}<s_{2}<\ldots<s_{\ell}$ be those values of $s \leq k$ where $\mathcal{W}_{j_{s}}^{(n)}(\mathbf{w})=1$, we deduce from the above equation that

$\sum_{r=1}^{\ell} \mathcal{Q}_{n}\left(\mathbf{v}, \mathcal{W}_{j_{s_{r}}}^{(n)}, u_{j_{s_{r}}}^{(n)}\right)=1 \quad$ on $\{\mathbf{v}\} \times\{\mathbf{w}\} \times \mathbb{Z}_{6} \times\left\{\mathbf{0}_{I_{1} \cup \ldots \cup I_{n-1} \cup\left\{y_{1}, \ldots, y_{n-1}\right\}}\right\}$.

However, each of the functions $\mathcal{Q}_{n}\left(\mathbf{v}, \mathcal{W}_{j_{s_{r}}}^{(n)}, u_{j_{s_{r}}}^{(n)}\right)$ restricted to the set $\{\mathbf{v} \oplus \mathbf{w}\} \times$ $\mathbb{Z}_{6} \times\left\{\mathbf{0}_{I_{1} \cup \ldots \cup I_{n-1} \cup\left\{y_{1}, \ldots, y_{n-1}\right\}}\right\} \cong \mathbb{Z}_{6}$ is simply a rotated copy of the function $\mathcal{A}$, and we introduced this precisely so as to have the property that the constant function 1 cannot be made as a linear combination of its rotates. This gives the desired contradiction.

Recursion clause Suppose that $1 \leq n_{1}<n_{2}<\ldots<n_{\ell}=m$ with $\ell \geq 2$ are the values of $n$ for which $\mathcal{F}_{n}$ is nonempty. We will show that in this case the 
assumption that

$$
\sum_{j=1}^{\ell} \sum_{i=1}^{i_{n_{j}}} \mathcal{Q}_{n_{j}}\left(\mathbf{u}_{i}^{\left(n_{j}\right)}, \mathcal{W}_{i}^{\left(n_{j}\right)}, u_{i}^{\left(n_{j}\right)}\right)=\eta \mathbf{1}_{\left\{\mathbf{u}^{\circ}\right\} \times \mathbb{Z}_{6}^{I_{1} \cup \cdots \cup I_{m} \cup\left\{y_{1}, \ldots, y_{m}\right\}}},
$$

implies that also

$$
\sum_{j=2}^{\ell} \sum_{i=1}^{i_{n_{j}}} \mathcal{Q}_{n_{j}}\left(\mathbf{u}_{i}^{\left(n_{j}\right)}, \mathcal{W}_{i}^{\left(n_{j}\right)}, u_{i}^{\left(n_{j}\right)}\right)=\eta \mathbf{1}_{\left\{\mathbf{u}^{\circ}\right\} \times \mathbb{Z}_{6}^{I_{1} \cup \cdots \cup I_{m} \cup\left\{y_{1}, \ldots, y_{m}\right\}}},
$$

(that is, omitting the first term of the outer sum) so that an induction on $\ell$ completes the proof.

To see this, observe that each of the functions $\mathcal{Q}_{n_{1}}\left(\mathbf{u}_{i}^{\left(n_{1}\right)}, \mathcal{W}_{i}^{\left(n_{1}\right)}, u_{i}^{\left(n_{1}\right)}\right)$ is supported on the set $\left\{\mathbf{u}_{i}^{\left(n_{1}\right)}\right\} \times \mathbb{Z}_{6}^{I_{n_{1}} \cup I_{n_{1}-1} \cup \ldots \cup I_{1} \cup\left\{y_{n_{1}}, y_{n_{1}-1}, \ldots, y_{1}\right\}}$, whereas each of the functions $\mathcal{Q}_{n_{j}}\left(\mathbf{u}_{i}^{\left(n_{j}\right)}, \mathcal{W}_{i}^{\left(n_{j}\right)}, u_{i}^{\left(n_{j}\right)}\right)$ for $j \geq 2$ is constant on every set of the form $\{\mathbf{v}\} \times \mathbb{Z}_{6}^{I_{n_{1}} \cup \cdots \cup I_{1} \cup\left\{y_{n_{1}}, \ldots, y_{1}\right\}}$ with $\mathbf{v} \in \mathbb{Z}_{6}^{\oplus G \backslash\left(I_{n_{1}} \cup \ldots \cup I_{1} \cup\left\{y_{n_{1}}, \ldots, y_{1}\right\}\right)}$, as is the func-

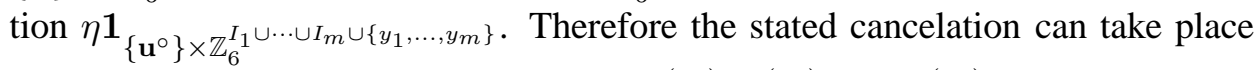
only if for every $\mathbf{v}$ that appears in the list $\mathbf{u}_{1}^{\left(n_{1}\right)}, \mathbf{u}_{2}^{\left(n_{1}\right)}, \ldots, \mathbf{u}_{i_{n_{1}}}^{\left(n_{1}\right)}$ we have

$\sum_{i \leq i_{n_{1}}, \mathbf{u}_{i}^{\left(n_{1}\right)}=\mathbf{v}} \mathcal{Q}_{n_{1}}\left(\mathbf{v}, \mathcal{W}_{i}^{\left(n_{1}\right)}, u_{i}^{\left(n_{1}\right)}\right)=$ const. $\quad$ on $\{\mathbf{v}\} \times \mathbb{Z}_{6}^{I_{n_{1}} \cup \cdots \cup I_{1} \cup\left\{y_{n_{1}}, \ldots, y_{1}\right\}}$,

and applying the argument for the base clause to this sub-sum tells us that the constant in question must be zero. Summing over $\mathbf{v}$ now gives

$$
\sum_{i=1}^{i_{n_{1}}} \mathcal{Q}_{n_{1}}\left(\mathbf{u}_{i}^{\left(n_{1}\right)}, \mathcal{W}_{i}^{\left(n_{1}\right)}, u_{i}^{\left(n_{1}\right)}\right)=0
$$

and finally subtracting this sum from the initially-given equation completes the recursion step, so the induction continues.

Remark If we assume that $\eta=0$ a priori then the above proposition asserts simply that the subspaces $U_{n}^{+} \leq \mathbb{Z}_{2}^{\oplus \mathbb{Z}_{6}^{\oplus G}}, n=1,2, \ldots$, are linearly independent over $\mathbb{Z}_{2}$. Indeed, given any equation

$$
\sum_{n=1}^{m} \sum_{i=1}^{i_{n}} \mathcal{Q}_{n}\left(\mathbf{u}_{i}^{(n)}, \mathcal{W}_{i}^{(n)}, u_{i}^{(n)}\right)=0
$$


we may first partition the left-hand side into sub-sums according to the values of $\left.\mathbf{u}_{i}^{(n)}\right|_{\left(G \backslash\left(I_{1} \cup \ldots \cup I_{m} \cup\left\{y_{1}, \ldots, y_{m}\right\}\right)\right)}$, and now these sub-sums must all vanish separately because any two maps $\mathcal{Q}_{n}\left(\mathbf{u}_{i}^{(n)}, \mathcal{W}_{i}^{(n)}, u_{i}^{(n)}\right)$ and $\mathcal{Q}_{n^{\prime}}\left(\mathbf{u}_{j}^{\left(n^{\prime}\right)}, \mathcal{W}_{j}^{\left(n^{\prime}\right)}, u_{j}^{\left(n^{\prime}\right)}\right)$ have disjoint support if these restrictions do not agree. Each of these sub-sums falls within the conditions of the proposition.

We are now ready to deduce the crucial relation between the metric $\operatorname{TS}_{\rho_{\left(\mathbb{Z}_{6} l^{\prime}\right)_{0}},\left(\mathbf{0}, e_{G}\right)}$ on $\mathbb{Z}_{2}^{\oplus\left(\mathbb{Z}_{6} \imath G\right)_{0}}$ and the metrics $\mathrm{TS}_{\mathrm{Ham}}$ on some of its finite-dimensional factor spaces.

Lemma 4.8. Provided the sequence of scales grows sufficiently fast, the metric $\mathrm{TS}_{\rho_{\left(\mathbb{Z}_{6} \imath G\right)_{0}},\left(\mathbf{0}, e_{G}\right)}$ admits the following kind of approximation. Suppose that $\mathcal{U}_{n}, \mathcal{V}_{n}$ : $\mathbb{Z}_{6}^{I_{n}} \rightarrow \mathbb{Z}_{2}$ are non-zero and distinct, and that $\mathcal{U}, \mathcal{V}: \mathbb{Z}_{6}^{\oplus G} \rightarrow \mathbb{Z}_{2}$ are such that

$$
\begin{aligned}
& \operatorname{spt} \mathcal{U} \subseteq\{\mathbf{0}\} \times \operatorname{spt} \mathcal{U}_{n} \times \mathbb{Z}_{6}^{\left(I_{1} \cup \cdots \cup I_{n-1} \cup\left\{y_{1}, \ldots, y_{n}\right\}\right)}, \\
& \text { spt } \mathcal{V} \subseteq\{\mathbf{0}\} \times \operatorname{spt} \mathcal{V}_{n} \times \mathbb{Z}_{6}^{\left(I_{1} \cup \cdots \cup I_{n-1} \cup\left\{y_{1}, \ldots, y_{n}\right\}\right)} \text {, }
\end{aligned}
$$

and

$$
\begin{aligned}
& \operatorname{spt} \mathcal{U} \cap\left(\{\mathbf{0}\} \times\{\mathbf{u}\} \times \mathbb{Z}_{6}^{\left(I_{1} \cup \cdots \cup I_{n-1} \cup\left\{y_{1}, \ldots, y_{n}\right\}\right)}\right) \neq \emptyset \quad \forall \mathbf{u} \in \operatorname{spt} \mathcal{U}_{n}, \\
& \operatorname{spt} \mathcal{V} \cap\left(\{\mathbf{0}\} \times\{\mathbf{u}\} \times \mathbb{Z}_{6}^{\left(I_{1} \cup \cdots \cup I_{n-1} \cup\left\{y_{1}, \ldots, y_{n}\right\}\right)}\right) \neq \emptyset \quad \forall \mathbf{u} \in \operatorname{spt} \mathcal{V}_{n} \\
& \left.\mathcal{U}\right|_{\{\mathbf{0}\} \times\{\mathbf{u}\} \times \mathbb{Z}_{6}^{\left(I_{1} \cup \cdots \cup I_{n-1} \cup\left\{y_{1}, \ldots, y_{n}\right\}\right)}}=\left.\mathcal{V}\right|_{\{\mathbf{0}\} \times\{\mathbf{u}\} \times \mathbb{Z}_{6}^{\left(I_{1} \cup \cdots \cup I_{n-1} \cup\left\{y_{1}, \ldots, y_{n}\right\}\right)}} \\
& \forall \mathbf{u} \in \operatorname{spt} \mathcal{U}_{n} \cap \operatorname{spt} \mathcal{V}_{n}
\end{aligned}
$$

Then we have

$$
r_{n} \cdot \operatorname{TS}_{\text {Ham }}\left(\mathcal{U}_{n}, \mathcal{V}_{n}\right) \leq \operatorname{TS}_{\rho_{\left(\mathbb{Z}_{6} \backslash G\right)_{0}},\left(\mathbf{0}, e_{G}\right)}(\mathcal{U}, \mathcal{V}) \leq 4 M r_{n} \cdot \operatorname{TS}_{\text {Ham }}\left(\mathcal{U}_{n}, \mathcal{V}_{n}\right)
$$

(irrespective of the actual sizes of the sets $\operatorname{spt} \mathcal{U}$, $\operatorname{spt} \mathcal{V}$ ).

Proof We now write simply $\mathbf{0}$ for the zero of either $\mathbb{Z}_{6}^{\oplus G}$ or $\mathbb{Z}_{6}^{\oplus G \backslash\left(I_{1} \cup \cdots \cup I_{n} \cup\left\{y_{1}, \ldots, y_{n}\right\}\right)}$ for any $n$, since among these possibilities the index set will always be clear from the context.

If the distance $r_{n}$ is sufficiently large compared with $r_{1}, \rho\left(e_{G}, y_{1}\right), r_{2}, \rho\left(e_{G}, y_{2}\right)$, $\ldots, r_{n-1}$ and $\rho\left(e_{G}, y_{n}\right)$, and if $\mathbf{u}, \mathbf{u}^{\prime} \in \mathbb{Z}_{6}^{I_{n}}$ are distinct, then the pinned traveling salesman distance in $\left(\mathbb{Z}_{6} \zeta G\right)_{0}$ between any points of the cylinder sets

$$
\{\mathbf{0}\} \times\{\mathbf{u}\} \times \mathbb{Z}_{6}^{\left(I_{1} \cup \cdots \cup I_{n-1} \cup\left\{y_{1}, \ldots, y_{n}\right\}\right)}
$$


and

$$
\{\mathbf{0}\} \times\left\{\mathbf{u}^{\prime}\right\} \times \mathbb{Z}_{6}^{\left(I_{1} \cup \cdots \cup I_{n-1} \cup\left\{y_{1}, \ldots, y_{n}\right\}\right)}
$$

will be

$c \cdot \rho_{\left(\mathbb{Z}_{6} \imath G\right)_{0}}\left(\mathbf{0} \oplus \mathbf{u} \oplus \mathbf{0}_{I_{1} \cup \ldots \cup I_{n-1} \cup\left\{y_{1}, \ldots, y_{n}\right\}}, \mathbf{0} \oplus \mathbf{u}^{\prime} \oplus \mathbf{0}_{I_{1} \cup \ldots \cup I_{n-1} \cup\left\{y_{1}, \ldots, y_{n}\right\}}\right) \geq r_{n}$,

for some $1 \leq c \leq 2$, since the length of a cycle in $G$ needed to cover all the coordinates where $\mathbf{u}$ differs from $\mathbf{u}^{\prime}$ dwarfs the maximum number of steps that could possibly be needed to cover all the differences between two points necessary at coordinates indexed by $I_{1} \cup \cdots \cup I_{n-1} \cup\left\{y_{1}, \ldots, y_{n}\right\}$.

Similarly, if $r_{n}$ is sufficiently large then the pinned traveling salesman distance between these cylinder sets also dwarfs the maximum pinned traveling salesman length of any non-self-intersecting path within either of these cylinder sets.

It follows for $\mathcal{U}$ and $\mathcal{V}$ as given that any cycle in $\left(\mathbb{Z}_{6} \zeta G\right)_{0}$ starting and ending at $\left(\mathbf{0}, e_{G}\right)$ and covering $\operatorname{spt}(\mathcal{U}+\mathcal{V})$ needs to cover at least one point of each of the sets

$$
\operatorname{spt} \mathcal{U} \cap\left(\{\mathbf{0}\} \times\{\mathbf{u}\} \times \mathbb{Z}_{6}^{\left(I_{1} \cup \cdots \cup I_{n-1} \cup\left\{y_{1}, \ldots, y_{n}\right\}\right)}\right) \quad \text { for } \mathbf{u} \in \operatorname{spt} \mathcal{U}_{n} \backslash \operatorname{spt} \mathcal{V}_{n}
$$

and

$$
\operatorname{spt} \mathcal{V} \cap\left(\{\mathbf{0}\} \times\{\mathbf{u}\} \times \mathbb{Z}_{6}^{\left(I_{1} \cup \cdots \cup I_{n-1} \cup\left\{y_{1}, \ldots, y_{n}\right\}\right)}\right) \quad \text { for } \mathbf{u} \in \operatorname{spt} \mathcal{V}_{n} \backslash \operatorname{spt} \mathcal{U}_{n}
$$

but no others, and that the distance between any two of these sets is much larger than the maximum number of steps in a path that could be needed to cover the necessary points within any one of them.

Therefore the length of such a covering traveling salesman cycle for $\operatorname{spt}(\mathcal{U}+\mathcal{V})$ is bounded below by the length of a traveling salesman cycle in $\left(\mathbb{Z}_{6} \zeta G\right)_{0}$ for the set

$$
\left\{\left.\mathbf{0} \oplus \mathbf{u} \oplus \mathbf{0}\right|_{I_{1} \cup \ldots \cup I_{n-1} \cup\left\{y_{1}, \ldots, y_{n}\right\}}: \mathbf{u} \in \operatorname{spt} \mathcal{U}_{n} \triangle \operatorname{spt} \mathcal{V}_{n}\right\},
$$

and if $r_{n}$ is sufficiently large compared with its predecessors then it may also be bounded from above by twice this number. Finally, since by construction any two index points of $I_{n}$ are separated by a $\rho$-distance that lies in $\left[r_{n}, 2 M r_{n}\right]$, and so any covering cycle for this latter set must traverse an additional distance of at least $r_{n}$ and at most $2 M r_{n}$ for each new point that it must visit, we deduce this latter distance lies between $r_{n} \cdot \mathrm{TS}_{\mathrm{Ham}}\left(\mathcal{U}_{n}, \mathcal{V}_{n}\right)$ and $2 M r_{n} \cdot \mathrm{TS}_{\mathrm{Ham}}\left(\mathcal{U}_{n}, \mathcal{V}_{n}\right)$.

Combining the bounds obtained above now completes the proof. 
Proof of Proposition 4.5 First, Lemma 4.6 allows us to consider instead the maps

$$
\lambda_{n}^{\circ}: \mathbb{Z}_{2}^{\mathbb{Z}_{6}^{I_{n}}} \rightarrow \mathbb{Z}_{2}^{\oplus\left(\mathbb{Z}_{6} \imath G\right)_{0}} / U: \mathcal{W} \mapsto \mathcal{Q}_{n}(\mathbf{0}, \mathcal{W}, 0)+U,
$$

for which it will suffice to show the corresponding properties:

1. $\lambda_{n}^{\circ}$ is $\mathbb{Z}_{2}$-linear with kernel $D_{n}^{\perp}$;

2. the resulting quotient map

$$
\lambda_{n}: \mathbb{Z}_{2}^{\mathbb{Z}_{6}^{I_{n}}} / D_{n}^{\perp} \hookrightarrow \mathbb{Z}_{2}^{\oplus\left(\mathbb{Z}_{6} \curlywedge G\right)_{0}} / U
$$

is a bi-Lipschitz embedding of $\mathrm{TS}_{\mathrm{Ham} / D_{n}^{\perp}}$ into $\operatorname{TS}_{\rho_{\left(\mathbb{Z}_{6} \ell G\right)_{0}},\left(\mathbf{0}, e_{G}\right) / U}$ with distortion at most $\mathrm{O}(M)$ and expansion ratio $\mathrm{O}\left(r_{n}\right)$.

1. It is clear from the definition of $U_{n} \leq U$ that $\lambda_{n}^{\circ}$ annihilates $D_{n}^{\perp}$, so we need only show that it does not annihilate any larger subspace of $\mathbb{Z}_{2}^{\mathbb{Z}_{6}}$. However, a special case of Lemma 4.7 tells us that the subspaces $U_{n}^{+} \leq \mathbb{Z}_{2}^{\oplus\left(\mathbb{Z}_{6} \zeta G\right)_{0}}$ are linearly independent (as remarked immediately after that lemma). It follows that if $\lambda_{n}^{\circ}(\mathcal{V})=\mathcal{Q}_{n}(\mathbf{0}, \mathcal{V}, 0)+U$ is equal to the identity element $U$ in $\mathbb{Z}_{2}^{\oplus\left(\mathbb{Z}_{6} \imath G\right)_{0}} / U$, then there are elements $\mathcal{A}_{1} \in U_{1}, \mathcal{A}_{2} \in U_{2}, \ldots, \mathcal{A}_{m} \in U_{m}$ for some $m \geq n$ such that

$$
\mathcal{A}_{1}+\mathcal{A}_{2}+\cdots+\left(\mathcal{Q}_{n}(\mathbf{0}, \mathcal{V}, 0)+\mathcal{A}_{n}\right)+\cdots+\mathcal{A}_{m}=\mathbf{0},
$$

and this is possible only if each $\mathcal{A}_{i}$ is individually zero for $i \neq n$ and also $\mathcal{Q}_{n}(\mathbf{0}, \mathcal{V}, 0)=$ $\mathcal{A}_{n}$.

We now express $\mathcal{A}_{n}$ as

$$
\sum_{i=1}^{i_{1}} \mathcal{Q}_{n}\left(\mathbf{u}_{i}, \mathcal{W}_{i}, u_{i}\right) \quad \mathcal{W}_{i} \in D_{n}^{\perp}, u_{i} \in \mathbb{Z}_{6} \quad \forall i \leq i_{1},
$$

and will show that we can have $\mathcal{Q}_{n}(\mathbf{0}, \mathcal{V}, 0)$ equal to such a sum only if in fact $\mathcal{V}$ is itself a member of $D_{n}^{\perp}$. First, we may clearly discard all terms of this sum for which $\mathbf{u}_{i} \neq \mathbf{0}$, since Lemma 4.7 tells us immediately that these must cancel to 0 , and relabel so that $\mathbf{u}_{i}=\mathbf{0}$ for every $i \leq i_{1}$. Therefore, recalling the definition of $\mathcal{Q}_{n}$ and omitting the fixed vector $\mathbf{0}$, the above equation becomes

$$
\mathcal{V}(\mathbf{w}) \cdot \mathcal{A}(w)=\sum_{i=1}^{i_{1}} \mathcal{W}_{i}(\mathbf{w}) \cdot \mathcal{A}\left(w-u_{i}\right) \quad \forall \mathbf{w} \in \mathbb{Z}_{6}^{I_{n}}, w \in \mathbb{Z}_{6}
$$


However, if we know simply fix $w \in \operatorname{spt} \mathcal{A}$, this equation simplifies to

$$
\mathcal{V}(\mathbf{w})=\sum_{1 \leq i \leq i_{1}, \mathcal{A}\left(w-u_{i}\right)=1} \mathcal{W}_{i}(\mathbf{w}) \quad \forall \mathbf{w} \in \mathbb{Z}_{6}^{I_{n}}
$$

and so we have expressed $\mathcal{V}$ as a linear combination of members of $D_{n}^{\perp}$, and hence proved that it itself lies in $D_{n}^{\perp}$, as required.

2. We prove the two necessary inequalities separately. First we prove that

$\operatorname{TS}_{\rho_{\left(\mathbb{Z}_{6} \succ G\right)_{0}},\left(\mathbf{0}, e_{G}\right) / U}\left(\lambda_{n}\left(\mathcal{U}+D_{n}^{\perp}\right), \lambda_{n}\left(\mathcal{V}+D_{n}^{\perp}\right)\right) \leq 4 M r_{n} \cdot \operatorname{TS}_{\text {Ham } / D_{n}^{\perp}}\left(\mathcal{U}+D_{n}^{\perp}, \mathcal{V}+D_{n}^{\perp}\right)$

Indeed, if $\mathcal{W} \in D_{n}^{\perp}$ minimizes $\operatorname{TS}_{\text {Ham }}(\mathcal{U}, \mathcal{V}+\mathcal{W})$, then $\mathcal{Q}_{n}(\mathbf{0}, \mathcal{W}, 0) \in U_{n} \leq U$ and by a simple application of Lemma 4.8 the distance

$$
\operatorname{TS}_{\rho_{\left(\mathbb{Z}_{6} \imath G\right)_{0}},\left(\mathbf{0}, e_{G}\right)}\left(\mathcal{Q}_{n}(\mathbf{0}, \mathcal{U}, 0), \mathcal{Q}_{n}(\mathbf{0}, \mathcal{V}, 0)+\mathcal{Q}_{n}(\mathbf{0}, \mathcal{W}, 0)\right)
$$

is at least $r_{n}$ and at most $4 M r_{n}$ times $\operatorname{TS}_{\mathrm{Ham}}(\mathcal{U}, \mathcal{V}+\mathcal{W})$, as required.

The necessary reverse inequality

$\operatorname{TS}_{\rho_{\left(\mathbb{Z}_{6} \ell G\right)_{0}},\left(\mathbf{0}, e_{G}\right) / U}\left(\lambda_{n}\left(\mathcal{U}+D_{n}^{\perp}\right), \lambda_{n}\left(\mathcal{V}+D_{n}^{\perp}\right)\right) \gtrsim r_{n} \cdot \operatorname{TS}_{\text {Ham } / D_{n}^{\perp}}\left(\mathcal{U}+D_{n}^{\perp}, \mathcal{V}+D_{n}^{\perp}\right)$

requires a little more work. By addition-invariance we may assume that $\mathcal{U}=\mathbf{0}$. Now suppose that $\mathcal{V} \in \mathbb{Z}_{2}^{\mathbb{Z}_{6}^{I_{n}}}$ and $m \geq n$ and that

$$
\begin{array}{r}
\mathcal{F}_{k}:=\left\{\mathcal{Q}_{k}\left(\mathbf{u}_{1}^{(k)}, \mathcal{W}_{1}^{(k)}, u_{1}^{(k)}\right), \mathcal{Q}_{k}\left(\mathbf{u}_{2}^{(k)}, \mathcal{W}_{2}^{(k)}, u_{2}^{(k)}\right), \ldots, \mathcal{Q}_{k}\left(\mathbf{u}_{i_{k}}^{(k)}, \mathcal{W}_{i_{k}}^{(k)}, u_{i_{k}}^{(k)}\right)\right\} \\
\text { for } k=1,2, \ldots, m
\end{array}
$$

are finite collections in each $U_{k}$. Clearly we may assume in our notation that the functions $\mathcal{W}_{i}^{(k)}$ are all non-zero, that $\mathcal{F}_{m} \neq \emptyset$ and finally that $m$ is minimal subject to all these other restrictions.

To complete the proof we will show that for some such finite data the combined collection $\mathcal{F}:=\bigcup_{k \leq m} \mathcal{F}_{k}$ actually minimizes the value

$$
\operatorname{TS}_{\rho_{\left(\mathbb{Z}_{6} \imath G\right)_{0}},\left(\mathbf{0}, e_{G}\right)}\left(\mathbf{0}, \mathcal{Q}_{n}(\mathbf{0}, \mathcal{V}, 0)+\Sigma \mathcal{F}\right)
$$

over all such finite tuples of finite collections, where we write $\Sigma \mathcal{F}$ simply for the sum in $\mathbb{Z}_{2}^{\oplus\left(\mathbb{Z}_{6} \zeta G\right)_{0}}$ of all elements of $\mathcal{F}$, and then from this minimizing $\mathcal{F}$ we will construct some $\mathcal{W} \in D_{n}^{\perp}$ so that

$$
\operatorname{TS}_{\rho_{\left(\mathbb{Z}_{6} \succ G\right)_{0}},\left(\mathbf{0}, e_{G}\right)}\left(\mathbf{0}, \mathcal{Q}_{n}(\mathbf{0}, \mathcal{V}, 0)+\Sigma \mathcal{F}\right) \geq r_{n} \cdot \operatorname{TS}_{\mathrm{Ham}}(\mathbf{0}, \mathcal{V}+\mathcal{W})
$$


Step (i) We first show by contradiction that in order to infimize the expression (2) over finite tuples $\mathcal{F}_{k}, k \leq m$, it suffices to assume that $m \leq n$. Indeed, suppose instead that $m \geq n+1$. First, if any $\mathbf{u}_{i}^{(m)}$ is not $\mathbf{0}$, then we may simply omit the corresponding member of $\mathcal{F}_{m}$ together with all members of any $\mathcal{F}_{k}$ with $k<m$ whose supports it dominates, and obtain a new family $\mathcal{F}^{\prime}$ for which

$\operatorname{TS}_{\rho_{\left(\mathbb{Z}_{6} \backslash G\right)_{0}},\left(\mathbf{0}, e_{G}\right)}\left(\mathbf{0}, \mathcal{Q}_{n}(\mathbf{0}, \mathcal{V}, 0)+\Sigma \mathcal{F}^{\prime}\right)<\operatorname{TS}_{\rho_{\left(\mathbb{Z}_{6} \backslash G\right)_{0}},\left(\mathbf{0}, e_{G}\right)}\left(\mathbf{0}, \mathcal{Q}_{n}(\mathbf{0}, \mathcal{V}, 0)+\Sigma \mathcal{F}\right)$

Therefore it suffices to assume that $\mathbf{u}_{i}^{(m)}=\mathbf{0}$ for all $i \leq i_{m}$. Next, for any given $w \in \mathbb{Z}_{6}$, we have

$$
\begin{array}{r}
\sum_{i \leq i_{m}} \mathcal{Q}_{m}\left(\mathbf{0}, \mathcal{W}_{i}^{(m)}, u_{i}^{(m)}\right)(\mathbf{0} \oplus \mathbf{w} \oplus w \oplus \mathbf{u})=\sum_{i \leq i_{m}, \mathcal{A}\left(w-u_{i}^{(m)}\right)=1} \mathcal{W}_{i}^{(m)}(\mathbf{w}) \\
\text { for } \mathbf{w} \in \mathbb{Z}_{6}^{I_{m}}, \mathbf{u} \in \mathbb{Z}_{6}^{I_{1} \cup \ldots \cup I_{m-1} \cup\left\{y_{1}, \ldots, y_{m-1}\right\}} .
\end{array}
$$

Regarded as a function of $\mathbf{w} \in \mathbb{Z}_{6}^{I_{m}}$ alone, we see that this is a member of $D_{m}^{\perp}$. If it is zero for every $w \in \mathbb{Z}_{6}$, then summing over $w$ shows that $\Sigma \mathcal{F}_{m}=0$, and so we may simply discard $\mathcal{F}_{m}$ to leave a new family $\mathcal{F}^{\prime}:=\bigcup_{k \leq m-1} \mathcal{F}_{k}$ that achieves the same value for the expression (2) but has a smaller value of $m$. Hence after making finitely many such omissions, we may assume also that there is some $w \in \mathbb{Z}_{6}$ for which the above sum specifies a nonzero member of $D_{m}^{\perp}$; let us call that member $\mathcal{Y}$. Then we also have $\mathcal{Y} \neq \delta_{\mathbf{0}}$ (simply because $\delta_{\mathbf{0}} \notin D_{m}^{\perp}$ ), and so to this $w$ there corresponds some $\mathbf{w} \in \mathbb{Z}_{6}^{I_{m}} \backslash\{\mathbf{0}\}$ for which $\mathcal{Y}(\mathbf{w}) \neq 0$.

However, it now follows that the function $\sum_{i \leq i_{m}} \mathcal{Q}_{m}\left(\mathbf{0}, \mathcal{W}_{i}^{(m)}, u_{i}^{(m)}\right)$ is non-zero on the cylinder $\{\mathbf{0}\} \times\{\mathbf{w}\} \times\{w\} \times \mathbb{Z}_{6}^{I_{1} \cup \cdots \cup I_{m-1} \cup\left\{y_{1}, \ldots, y_{m-1}\right\}}$, and so actually takes the constant value 1 on that cylinder. By Lemma 4.7 the sum function $\mathcal{Q}_{n}(\mathbf{0}, \mathcal{V}, 0)+\Sigma\left(\mathcal{F}_{1} \cup \cdots \cup \mathcal{F}_{m-1}\right)$ cannot be constant and equal to 1 on this cylinder, and so overall the function $\mathcal{Q}_{n}(\mathbf{0}, \mathcal{V}, 0)+\Sigma \mathcal{F}$ does not vanish on this cylinder. On the other hand, if our sequence of scales grows fast enough, then any point of this cylinder is by itself much further away from $\left(\mathbf{0}, e_{G}\right)$ in $\rho_{\left(\mathbb{Z}_{6} \ell G\right)_{0}}$ than any possible value of $\operatorname{TS}_{\rho_{\left(\mathbb{Z}_{6}{ }^{\prime} G\right)_{0},\left(\mathbf{0}, e_{G}\right)}}\left(\mathbf{0}, \mathcal{Q}_{n}(\mathbf{0}, \mathcal{V}, 0)\right)$, and so in this case even the empty family gives a smaller value for the expression (2) than does $\mathcal{F}$. Therefore the infimum of the values (2) is unchanged if we only infimize over families of collections $\mathcal{F}$ for which $m \leq n$.

Step (ii) Having reduced to the case $m \leq n$, we can now argue as above to reduce the evaluation of the infimum of (2) further to the case when

$$
\left.\mathbf{u}_{i}^{(k)}\right|_{G \backslash\left(I_{1} \cup \cdots \cup I_{n} \cup\left\{y_{1}, \ldots, y_{n}\right\}\right)}=\mathbf{0}_{G \backslash\left(I_{1} \cup \cdots \cup I_{n} \cup\left\{y_{1}, \ldots, y_{n}\right\}\right)} \quad \forall k \leq m,
$$


since any of the functions in question is either supported on the cylinder specified by this collection of 0 -valued coordinates or is identically 0 on it, and so discarding those that are identically zero gives another collection $\mathcal{F}$ for which the value (2) is not any larger. This last reduction leaves only finitely many possibilities for the collection $\mathcal{F}$, and so now we know that we may pick one that is actually minimizing. One last 'processing' step will give us the construction of $\mathcal{W}$ from it.

Step (iii) For this, it now follows from another application of Lemma 4.7 that on any of the cylinders $\{\mathbf{0}\} \times\{\mathbf{w}\} \times\{0\} \times \mathbb{Z}_{6}^{I_{1} \cup \cdots \cup I_{n-1} \cup\left\{y_{1}, \ldots, y_{n-1}\right\}}$ for $\mathbf{w} \in \mathbb{Z}_{6}^{I_{n}}$ the sum function $\mathcal{Q}_{n}(\mathbf{0}, \mathcal{V}, 0)+\Sigma \mathcal{F}$ can vanish only if each individual sum $\Sigma \mathcal{F}_{k}$ vanishes there for each $k \leq n-1$, and also $\mathcal{Q}_{n}(\mathbf{0}, \mathcal{V}, 0)+\Sigma \mathcal{F}_{n}$ vanishes there. Substituting the definition of $\mathcal{Q}_{n}$ into this latter condition, it becomes that

$$
\mathcal{V}(\mathbf{w})+\sum_{1 \leq i \leq i_{n}, \mathcal{A}\left(-u_{i}^{(n)}\right)=1} \mathcal{W}_{i}^{(n)}(\mathbf{w})=0
$$

Therefore, the indicator function of the set

$$
\left\{\mathbf{w} \in \mathbb{Z}_{6}^{I_{n}}:\left.\left(\mathcal{Q}_{n}(\mathbf{0}, \mathcal{V}, 0)+\Sigma \mathcal{F}\right)\right|_{\{\mathbf{0}\} \times\{\mathbf{w}\} \times\{0\} \times \mathbb{Z}_{6}^{I_{1} \cup \cdots \cup I_{n-1} \cup\left\{y_{1}, \ldots, y_{n-1}\right\}}} \not \equiv 0\right\}
$$

is precisely $\mathcal{V}+\sum_{1 \leq i \leq i_{n}, \mathcal{A}\left(-u_{i}^{(n)}\right)=1} \mathcal{W}_{i}^{(n)}$, and therefore it is a member of $\mathcal{V}+D_{n}^{\perp}$; let us call it $\mathcal{V}+\mathcal{W}$. By Lemma 2.5 and Lemma4.8 this now implies at once that

$$
\operatorname{TS}_{\rho_{\left(\mathbb{Z}_{6}{ }^{2}\right)_{0}},\left(\mathbf{0}, e_{G}\right)}\left(\mathbf{0}, \mathcal{Q}_{n}(\mathbf{0}, \mathcal{V}, 0)+\Sigma \mathcal{F}\right) \geq r_{n} \cdot \operatorname{TS}_{\mathrm{Ham}}(\mathbf{0}, \mathcal{V}+\mathcal{W})
$$

as required.

\section{Further questions}

I suspect that the construction above can be extended so far as to give a finitelygenerated amenable group $G$ with a word metric $\rho$ for which any 1-Lipschitz embedding $f: G \hookrightarrow L_{p}, p \in[1, \infty)$, must be such that

$$
\|f(g)-f(h)\| \lesssim \log \rho(g, h)
$$

for some collection of pairs $g, h \in G$ among which $\rho(g, h)$ can be arbitrarily large.

Question Does every finitely-generated amenable group $G$ admit Lipschitz embeddings $f: G \hookrightarrow L_{p}$ for every $p \in[1, \infty)$ such that $\|f(g)-f(h)\|_{p} \gtrsim \log \rho(g, h)$ for all $g, h \in G$ ? 
Applied with a little less violence (that is, with $\eta<1$ in Lemma 3.1), the methods of this paper may also be useful for finding examples of finitely-generated amenable groups with specified compression exponents in $(0,1)$, although of course these methods will need to be complemented with matching lower-bound proofs for the groups in question (that is, constructions of particular good-distortion embeddings, on which the ideas of the present paper do not seem to bear directly). The kind of construction used above may also provide interesting test-cases for Question 10.6 of Naor and Peres [16], which asks for more general methods for estimating compression exponents of semidirect products; and on the question of which values are realizable as Euclidean compression exponents for finitely-generated amenable groups, specializing the result of Arzhantseva, Drutu and Sapir in [2] that all values in $[0,1]$ can be realized as the compression exponents of finitelygenerated groups that are not necessarily amenable.

It is interesting to note that in [12] Khot and Naor use inequalities described in terms of random walks on Hamming cubes to prove Theorem 3.2. Thus, although the random walk upper bound on compression exponents given by Naor and Peres cannot reach the zero-compression-exponent regime of the group $\left(\mathbb{Z}_{2}^{\oplus H} / V\right) \rtimes H$ constructed above, it seems that a modification of their method in which we allow ourselves to consider a sequence of random walks on our group supported on the images of these embedded finite cube-quotients would translate into the correct zero upper bound on the compression exponent. It would be interesting to find an example of a finitely-generated amenable group for which some other obstruction to embeddings with positive compression exponents is needed, genuinely unrelated to inequalities concerning random walks.

One candidate for such an obstruction would be a sequence of embedded copies of the cubes $\left(\mathbb{Z}_{2 m}^{n}, \ell_{\infty}\right)$ : given an embedded sequence of these with expansion ratios not growing too fast, we could instead use an analog of Lemma 3.1 based on the notion of non-linear cotype introduced by Mendel and Naor in [14], which is characterized in terms of these finite spaces. Given a suitable tradeoff between the sizes and expansion ratios, the arguments leading to Mendel and Naor's Theorem 1.11 of [14] would imply that such a sequence of embedded $\ell_{\infty}$-cubes obstructs good-compression embeddings into any Banach space with nontrivial type and cotype $q<\infty$, provided in addition that the parameter $m$ of the embedded cubes can be chosen to grow faster than $n^{1 / q}$. (Note also that whether the assumption of nontrivial type is necessary is one of the major outstanding problems from their paper.) However, at this stage I do not see how to construct a finitely-generated group that contains copies of these $\ell_{\infty}$-cubes with long side-lengths.

Finally, we remark that the methods above give a group with poor compression 
exponent specifically for embeddings into $L_{p}$ for $p<\infty$, because these are the Banach spaces to which Khot and Naor's analysis of cube-quotients in [12] applies (see Remark 3.1 of their paper). It is natural to ask whether some more 'purely geometric' feature of the choice of Banach target could be responsible for this poor embeddability.

Question Do the groups $\left(\mathbb{Z}_{2}^{\oplus H} / V\right) \ltimes H$ admit Lipschitz embeddings with positive compression exponents into any Banach space with finite cotype?

\section{References}

[1] I. Aharoni, B. Maurey, and B. S. Mityagin. Uniform embeddings of metric spaces and of Banach spaces into Hilbert spaces. Israel J. Math., 52(3):251265, 1985.

[2] G. Arzhantseva, C. Druţu, and M. Sapir. Compression functions of uniform embeddings of groups into Hilbert and Banach spaces. J. Reine Angew. Math., 633:213-235, 2009.

[3] G. N. Arzhantseva, V. S. Guba, and M. V. Sapir. Metrics on diagram groups and uniform embeddings in a Hilbert space. Comment. Math. Helv., 81(4):911-929, 2006.

[4] T. Austin, A. Naor, and Y. Peres. The wreath product of $\mathbb{Z}$ with $\mathbb{Z}$ has Hilbert compression exponent $\frac{2}{3}$. Proc. Amer. Math. Soc., 137(1):85-90, 2009.

[5] M. E. B. Bekka, P.-A. Cherix, and A. Valette. Proper affine isometric actions of amenable groups. In Novikov conjectures, index theorems and rigidity, Vol. 2 (Oberwolfach, 1993), volume 227 of London Math. Soc. Lecture Note Ser., pages 1-4. Cambridge Univ. Press, Cambridge, 1995.

[6] Y. de Cornulier, Y. Stalder, and A. Valette. Proper actions of wreath products and generalizations. Preprint.

[7] Y. de Cornulier, Y. Stalder, and A. Valette. Proper actions of lamplighter groups associated with free groups. C. R. Math. Acad. Sci. Paris, 346(34):173-176, 2008.

[8] Y. de Cornulier, R. Tessera, and A. Valette. Isometric group actions on Hilbert spaces: growth of cocycles. Geom. Funct. Anal., 17(3):770-792, 2007. 
[9] M. Gromov. Asymptotic invariants of infinite groups. In Geometric group theory, Vol. 2 (Sussex, 1991), volume 182 of London Math. Soc. Lecture Note Ser., pages 1-295. Cambridge Univ. Press, Cambridge, 1993.

[10] E. Guentner and J. Kaminker. Exactness and uniform embeddability of discrete groups. J. London Math. Soc. (2), 70(3):703-718, 2004.

[11] P. W. Jones. Rectifiable sets and the traveling salesman problem. Invent. Math., 102(1):1-15, 1990.

[12] S. Khot and A. Naor. Nonembeddability theorems via Fourier analysis. Math. Ann., 334(4):821-852, 2006.

[13] S. Li. Compression bounds of wreath products. Preprint.

[14] M. Mendel and A. Naor. Metric cotype. Ann. of Math. (2), 168(1):247-298, 2008.

[15] A. Naor and Y. Peres. Embeddings of discrete groups and the speed of random walks. Int. Math. Res. Not. IMRN, pages Art. ID rnn 076, 34, 2008.

[16] A. Naor and Y. Peres. $L_{p}$ compression, traveling salesmen and stable walks. Preprint, available online through http://www. cims.nyu.edu/ naor/, 2009.

[17] R. Tessera. Asymptotic isoperimetry on groups and uniform embeddings into Banach spaces. To appear, Comment. Math. Helv.

[18] R. Tessera. On the $l^{p}$ distortion of finite quotients of amenable groups. Preprint, available online at arXiv . org: 0706.3971, 2007.

[19] R. Tessera. Quantitative property A, Poincaré inequalities, $L^{p}$-compression and $L^{p}$-distortion for metric measure spaces. Geom. Dedicata, 136:203-220, 2008 .

[20] R. Tessera. Coarse embeddings into a Hilbert space, Haagerup property and Poincaré inequalities. J. Topol. Anal., 1(1):87-100, 2009.

[21] G. Yu. The coarse Baum-Connes conjecture for spaces which admit a uniform embedding into Hilbert space. Invent. Math., 139(1):201-240, 2000.

Department of Mathematics, Brown University, Box 1917, 151 Thayer Street, PROVIDENCE, RI 09212, USA

Email: timaustin@math.brown .edu

URL: http: / / www. math. brown. edu/ ${ }^{\text {timaustin }}$ 